\title{
Does Entropy Manufacture Impacts DM Density Profiles and How Well Does the Scientific Community Understand If or Not Gravity Is always Either a Classical and/or Quantum Phenomenon at Its Genesis over 13.7 Billion Years Ago?
}

\author{
Andrew Walcott Beckwith \\ Physics Department, College of Physics, Chongqing University Huxi Campus, Chongqing, China \\ Email: abeckwith@uh.edu, rwill9955b@gmail.com
}

\begin{abstract}
How to cite this paper: Beckwith, A.W. (2017) Does Entropy Manufacture Impacts DM Density Profiles and How Well Does the Scientific Community Understand If or Not Gravity Is always Either a Classical and/or Quantum Phenomenon at Its Genesis over 13.7 Billion Years Ago? Journal of High Energy Physics, Gravitation and Cosmology, 3, 106-137.
\end{abstract}

http://dx.doi.org/10.4236/jhepgc.2017.31014

Received: January 18, 2016

Accepted: January 21, 2017

Published: January 24, 2017

Copyright $\odot 2017$ by author and Scientific Research Publishing Inc. This work is licensed under the Creative Commons Attribution International License (CC BY 4.0). http://creativecommons.org/licenses/by/4.0/ Open Access

\begin{abstract}
In the 12th Marcel Grossmann Meeting, July $9^{\text {th }}, 2009$, the author raised the issue of whether early graviton production could affect non-Gaussian contributions to DM density profiles. Specifically, does a first-order phase transition, in the formation of GW also lead to variation in density fluctuations of space plasma production? and curvature perturbations? We submit that the answer to this question will lead to quantifying fluctuations in space time which affect the stability and formation of DM halos and DM density profiles. Furthermore, we look at whether or not there is a relationship between DM and DE, and gravitons. This is suggested by a modification of Randal Sundrum brane world models, which may be used to admit a very small four-dimensional standard space time non-zero graviton mass. Non zero graviton mass in 4 dimensional space time, as well as modification of existing KK graviton theories will lead to a speed-up of cosmological expansion when the red shift was approximately $\mathrm{z} \approx 0.5-0.55$, i.e., about a billion years ago. Finally, the issue of if gravity is a quantum phenomenon will be brought up in the context of understanding if or not squeezing of coherent states is mandatory at the onset of inflation.
\end{abstract}

\section{Keywords}

KK Graviton Theories, Non-Zero Graviton Mass,

Non-Gaussian Contributions to DM Density Profiles

\section{Introduction}

We wish to study how relic gravitational waves relate to relic gravitons [1], in order to 
answer some basic questions as to the likelihood of detection of GW, using appropriate instrumentation. To achieve this, we will examine some of the startling similarities and differences between GW equation dynamics and Kaluza-Klein (KK) gravitons [2]. This issue of apparently combined sources of planar wave generation of gravitational waves is a precursor to what would happen if squeezed states occurred in the onset of the big bang. i.e. what would happen with multiple super position of different coherent states? Part of what happens is that squeezed states in the beginning of inflation may be similar to multiple vacuum states contributing to different coherent states. Thereby introducing, at the beginning of inflation non Gaussian contributions to the initial relic gravitational wave forms. The sticking point is that, GWs are composed of coherent states of many gravitons [3], and coherent state of gravitons requires minimization of uncertainty and as in the simple harmonic oscillator (SHO), small deviations at best from semi-classical approximations. In the case of relic conditions, at the onset of inflation, there are many contributing vacuum states [4], which would make minimization of uncertainty highly problematic. However, the author suggests (for reasons to be presented in this paper) that gravitons from non-relic conditions may conceivably be measurable, although with difficulty. Recently [5] a PRD paper (Dr. Li et al., 2009) suggests a suitable GW measurement protocol, which may identify relic GW and show whether or not the conditions for graviton measurement are obtainable from astrophysical sources., provided an adequate detector is used. Li's PRD article [5] assumes a detector which measures the influx of gravitons from these astrophysical sources directly. The reproduced Table 1 presents a generally accepted range of GW frequencies.

Table 1. Magnitude, sources, and top frequency values for HFGW (from Li et al. 2008) [5].

\begin{tabular}{|c|c|c|c|}
\hline Sources & Amplitude & frequency & Characteristics \\
\hline $\begin{array}{l}\text { HFGW in Quintessence } \\
\text { inflationary models }\end{array}$ & $h_{r m s} \sim 10^{-30}-10^{-32} / \sqrt{\mathrm{Hz}}$ & $v \sim 10^{9}-10^{10} \mathrm{~Hz}$ & Random background \\
\hline $\begin{array}{l}\text { HFGW in some string } \\
\text { theory scenarios }\end{array}$ & $h_{r m s} \sim 10^{-30}-10^{-34} / \sqrt{\mathrm{Hz}}$ & $v \sim 10^{8}-10^{11} \mathrm{~Hz}$ & Random background \\
\hline Solar Plasma & $h_{r m s} \sim 10^{-39} / \sqrt{\mathrm{Hz}}$ & $v \sim 10^{15} \mathrm{~Hz}$ & On the Earth \\
\hline $\begin{array}{l}\text { High energy particles, } \\
\text { e.g. Fermi Ring }\end{array}$ & $h_{r m s} \sim 10^{-39}-10^{-41} / \sqrt{\mathrm{Hz}}$ & $v \sim 10^{4}-10^{5} \mathrm{~Hz}$ & $\begin{array}{l}\text { On the center the } \\
\text { frequency depends } \\
\text { upon the rotational } \\
\text { frequency of particles } \\
\text { in the Fermi Ring }\end{array}$ \\
\hline $\begin{array}{l}\text { Stanford Linear } \\
\text { Accelerator }\end{array}$ & $h_{r m s} \sim 10^{-39} / \sqrt{H z}$ & $v \sim 10^{23} \mathrm{~Hz}$ & $\begin{array}{l}\text { On the collision center, } \\
\text { the frequency depends } \\
\text { upon the self-energy } \\
\text { and the Lorentz factor of } \\
\text { high energy } \mathrm{e}^{+} \mathrm{e}^{-} \text {beams }\end{array}$ \\
\hline $\begin{array}{c}\text { LHC-Large } \\
\text { Hadron collider }\end{array}$ & & & $\begin{array}{l}\text { Spectra of high } \\
\text { energy gravitons }\end{array}$ \\
\hline $\begin{array}{l}\text { Nano-piezo electric } \\
\text { crystal array, with size of } \\
\text { about } 100 \text { nanometers }\end{array}$ & $h_{r m s} \sim 10^{-28}-10^{-31} / \sqrt{H z}$ & $v \sim 10^{9}-10^{10} \mathrm{~Hz}$ & $\begin{array}{l}\text { On the wave zone with } \\
\text { an effective cross section } \\
\text { of or less than } 0.01 \\
\text { meters squared, for } \\
\text { gravitational radiation }\end{array}$ \\
\hline
\end{tabular}


This reproduced PRD table [5] is important since it suggests that relic GW, if properly measured, may be the first ones to await experimental verification. The PRD authors suggest that focusing on relic GW would be the most likely method of detecting gravitational waves, using the detector design specified in the paper.

\section{Review of Simple Models as to Gravitons as Either Due to Strings/Something Else}

The following is a review of some of the material .Beckwith presented at Rencontres De Blois [6], in June, 2009. The summary of concepts will reflect upon interpretation of either the classical and/or quantum foundations of gravitons/ GW. How Equation (0.5) can be used to formulate appropriate operations.

\section{A. Two alternative routes to generation of entropy}

We wish to present two alternative routes to generation of entropy. The first, is a counting algorithm, as an adaptation of Y.J. Ng's infinite quantum (modified Boltzmann's) statistics [7], whereas the second is referencing A. Glinka's research presentation on "graviton gas [8]" as a way of understanding a different perspective as to how to get a partition function for gravitons which is congruent to the Wheeler De Witt equation. Here are a few questions which are posed for the reader to think about.

1. Is each "particle count unit" as brought up by $\mathrm{Ng}$, equivalent to a brane-antibrane unit in brane treatments of entropy?

2. Is the change of entropy $\Delta S \approx \Delta N_{\text {gravitons }}$ ?

3. Is this graviton production scheme comparable to Glinka's quantum gas, from the Wheeler De Witt equation?

\section{B. Entropy generation via $\mathrm{Ng}^{\prime}$ s infinite quantum statistics (short review)}

We wish to understand the linkage between dark matter and gravitons. To consider just that, we look at the "size" of the nucleation space, $V . V$ for nucleation is HUGE. Graviton space $V$ for nucleation is tiny, well inside inflation/therefore, the log factor drops OUT of entropy $S$ if $V$ chosen properly for both Equation (1) and Equation (2). $\mathrm{Ng}$ 's [7] result begins with a modification of the entropy/partition function $\mathrm{Ng}$ used the following approximation of temperature and its variation with respect to a spatial parameter, starting with temperature $T \approx R_{H}^{-1}$ ( $R_{H}$ can be thought of as a representation of the region of space where we take statistics of the particles in question). Furthermore, assume that the volume of space to be analyzed is of the form $V \approx R_{H}^{3}$ and look at a preliminary numerical factor we shall call $N \sim\left(R_{H} / l_{P}\right)^{2}$, where the denominator is Planck's length (on the order of $10^{-35}$ centimeters). We also specify a "wavelength" parameter $\lambda \approx T^{-1}$. So the value of $\lambda \approx T^{-1}$ and of $R_{H}$ are approximately the same order of magnitude. Now this is how Jack Ng changes conventional statistics: he outlines how to get $S \approx N$, which with additional arguments we refine to be $S \approx\langle n\rangle$ (where $\langle n\rangle$ is graviton density). Begin with a partition function

$$
Z_{N} \sim\left(\frac{1}{N !}\right) \cdot\left(\frac{V}{\lambda^{3}}\right)^{N}
$$

This, according to $\mathrm{Ng}$, [7] leads to entropy of the limiting value of, if $S=\left(\log \left[Z_{N}\right]\right)$

$$
S \approx N \cdot\left(\log \left[V / N \lambda^{3}\right]+5 / 2\right) \underset{\text { Ng-infinite-Quantum-Statistics }}{\longrightarrow} N \cdot\left(\log \left[V / \lambda^{3}\right]+5 / 2\right) \approx N
$$


But $V \approx R_{H}^{3} \approx \lambda^{3}$, so unless $\mathrm{N}$ in Equation (0.2) above is about $1, \mathrm{~S}$ (entropy) would be $<0$, which is a contradiction. Now this is where Jack Ng introduces removing the N! term in Equation (1) above, i.e., inside the Log expression we remove the expression of $\mathrm{N}$ in Equation (2) above. The modification of Ng's entropy expression [9] is in the region of space time for which the general temperature dependent entropy Kolb and Turner expression breaks down. In particular, the evaluation of entropy we do via the modified $\mathrm{Ng}$ argument above is in regions of space time where $g$ before re heat is an unknown, unmeasurable number of degrees of freedom The Kolb and Turner entropy expression [9] 1991 has a temperature $T$ related entropy density which leads to that we are able to state total entropy as the entropy density time's space time volume $V_{4}$ with $g_{\text {re-heat }} \approx 1000$, while dropping to $g_{\text {electro-weakt }} \approx 100$ in the electro weak era. This value of the space time degrees of freedom, according to de Vega has reached a low of $g_{\text {today }} \approx 2-3$ today. We assert that Equation (2) above occurs in a region of space time before $g_{\text {re-heat }} \approx 1000$, so after re heating Equation (2) no longer holds, and we instead can look at [9]

$$
S_{\text {total }} \equiv S_{\text {Density }} \cdot V_{4}=\frac{2 \pi^{2}}{45} \cdot g \cdot T^{3} \cdot V_{4}
$$

where $T<10^{32} \mathrm{~K}$. We can compare Equation (1) and Equation (2), as how they stack up with Glinka's (2007) quantum gas [8], if we set $\Omega=\frac{1}{2|u|^{2}-1}$ as a partition function (with $u$ part of a Bogoliubov transformation) due to a graviton-quintessence gas, to get information theory based entropy

$$
S \equiv \ln \Omega
$$

Such a linkage would open up the possibility that the density of primordial gravitational waves could be examined, and linked to modeling gravity as an effective theory. The details of linking what is done with Equation (2) and bridging it to Equation (3) await additional theoretical development, and are probably conceptually understandable if the following is used to link the two regimes. i.e. we can use the number of space time operations used to create Equation (2), via Seth Lloyds [10]

$$
I=S_{\text {total }} / k_{B} \ln 2=[\# \text { operations }]^{3 / 4}=\left[\rho \cdot c^{5} \cdot t^{4} / \hbar\right]^{3 / 4}
$$

Essentially, what will be done is to use Equation (5) to show linkage between a largely thermally based production of entropy, as implied by Equation (3) and a particle counting algorithm, as given by Equation (2). This due to the problems inherent in making connections between a particle count generation of entropy, and thermal contributions. i.e. two different processes are involved.

\section{Introduction. Connection between gravitons and GWs}

The first topic to raise is whether or not there is a way to make a connection between gravitons and GWs. In perturbative string theory, a graviton is a closed string in a very particular low-energy vibrational state. And in string theory, a graviton can be connected to a gravitational wave by linking the graviton particle to the curvature of the space-time continuum and calculating the gravitational force exerted. Unfortunately, for string theory, the only way to link gravitons to GWs is by obtaining the coherent 
state of many gravitons, i.e., looking at Gaussian states with minimum uncertainty, which would be stationary However, as Grishchuck showed, as reported by Allen, Flanagan, and Papa [11] (1999) relic GW generation is Gaussian, but NOT stationary,... Now can a standard planar approximation optimally work for detecting GWs? Probably not, based on Ming-Lei Tong and Yang Zhang [12] (2007), using GW spectra and numerical simulations, which gave a null result for detector (circular waveguides). This result has already been established by Ingley and Criuse (2001) [13]. So, let us see how the inputs gravitational waves OF WHAT? to the circular wave guide via numerical representation of planar waves for GW/ Gravitons MEANING? was initiated.

To do this, we need to consider the behavior of relic GW, as suggested by Tong and Zhang [12] (2007): $h_{i j}(x, \tau)$ in $\mathrm{d} S^{2} \equiv a^{2}(\tau) \cdot\left[-\mathrm{d}^{2} \tau+\left(\delta^{i j}+h_{i j}\right) \cdot \mathrm{d} x^{i} \mathrm{~d} x^{j}\right]$, in flat space (neglecting curvature), [12]

$$
\partial_{\mu}=\left(\sqrt{g} \cdot \partial^{\mu} h_{i j}(x, \tau)\right)=0
$$

This has the very simple solution, with a mean average for the approximate square of $h_{i j}(x, \tau)$

$$
\left\langle h^{i j}(x, \tau) h_{i j}(x, \tau)\right\rangle=\int_{0}^{\infty} h^{2}\left(k, \tau_{H}\right) \cdot \frac{\mathrm{d} k}{k}
$$

where the spectrum $h\left(k, \tau_{H}\right)$ may be given via $k_{H}=2 \pi \gamma, \gamma$ as an "accelerating parameter," $k_{H}=2 \pi \gamma, k_{S} \cong 10^{26} k_{H}, \in \equiv(1+\beta) \cdot(1-\gamma) / \gamma$, with $\beta$ an inflation parameter, and $\beta_{S}$ a re-heating parameter, so that

$$
h\left(k, \tau_{H}\right) \cong A_{0}\left(\frac{k_{S}}{k_{H}}\right)^{\beta_{S}} \cdot \frac{k_{H}}{k_{2}} \cdot\left(\frac{k}{k_{H}}\right)^{\beta-\beta_{S+1}} \cdot \frac{1}{\left(1+Z_{E}\right)^{3+\epsilon}}
$$

This is where one can write

$$
k=v \cdot 2 \pi \cdot a\left(\tau_{H}\right)
$$

where $v$ is a physical frequency, $\beta$ as an inflation parameter, $\beta_{S}$ as a re-heating parameter, $1+Z_{E}=\frac{a\left(\tau_{H}\right)}{a\left(\tau_{E}\right)} \sim\left[\frac{\Omega_{\Lambda}}{\Omega_{E}}\right]^{1 / 3} \sim 1.5$, where the red shift $Z_{E}$ is defined roughly via the time of equality between dark energy and matter density, with $Z_{E}=0.5-0.55$ as given by Eric Linder [14] (2003): in PRL (rapid communications) in his Figure 1. We need to ask ourselves though whether or not the representation OF WHAT? make sense, because in the regime of billions of years after a big bang, it restricts us to making use of planar approximations to GW and attendant gravitons. Note that the approximation of gravitational spectra given by $(0.6 \mathrm{~b})$ leads to a null result in simulations of detectable relic GW by Tong and Zhang [12] (2007). So probably a more refined version of GW representation needs to be given. Now, can we connect the wavelength of a graviton and the GW frequency? Clifford Will [15] (1997) wrote the wavelength of a graviton as, $\lambda_{\text {Graviton }} \equiv\left(h / m_{\text {graviton }} \cdot c\right)$ while other authors have suggested $m_{\text {graviton }} \approx 10^{-65}$ grams. As Will observes, if $\mathrm{f}$ is the frequency, and the wavelength $\lambda_{\text {graviton }}$ may play a role in modified gravity, via an effective Newtonian (gravitational) potential of $V \propto\left[\exp \left(-r / \lambda_{\text {graviton }}\right)\right] / r$, i.e. are there observational ways to obtain a graviton wave length Will in (1997) [15] experimentally estimated the magnitude of $\lambda_{\text {graviton }} \sim 6 \times 10^{12}$ 


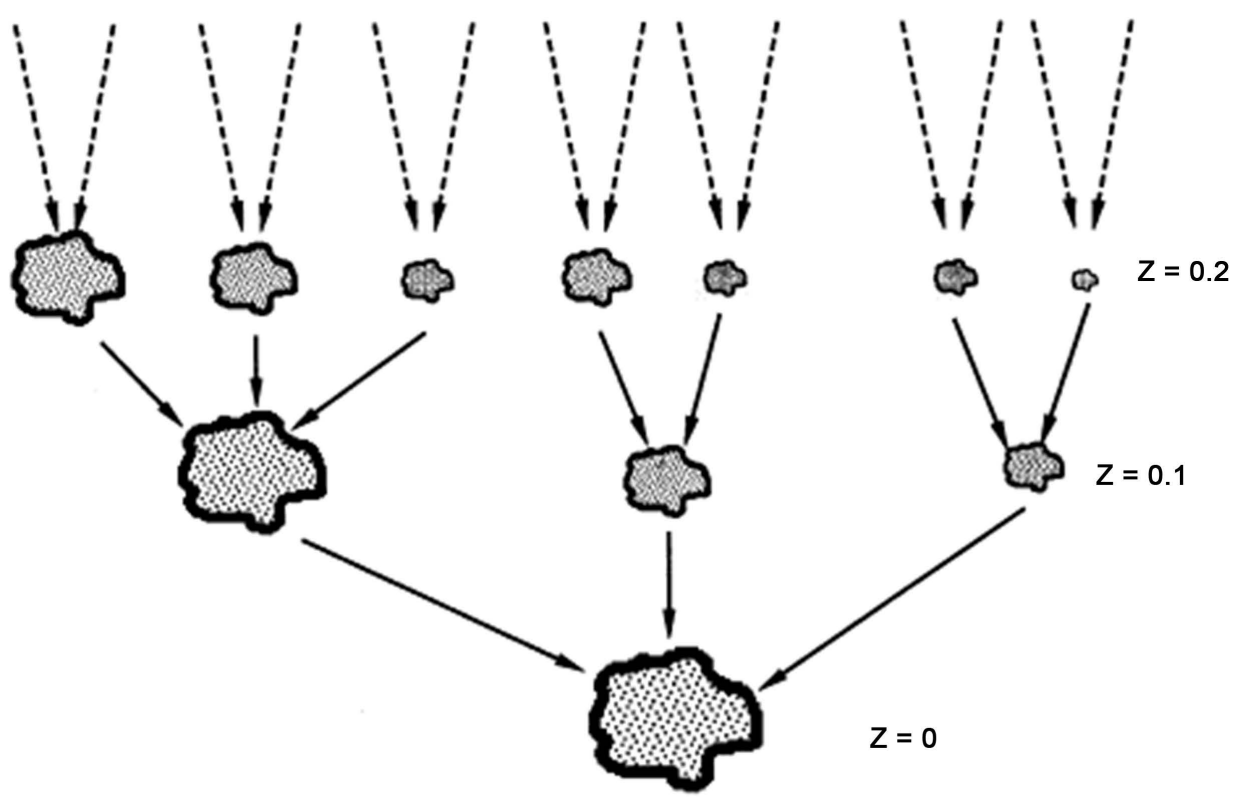

Figure 1. i.e. how we obtain from the "bottom up" development of galactic super structure [44].

kilometers, which would make modified gravitational measurements a near impossibility. i.e. one of the challenges would be to see if or not experimental protocol exists that would allow tests of

$$
[\text { velocity }]_{\text {graviton }} \equiv c \cdot \sqrt{\left(1-\left(c^{2} / f^{2} \lambda_{\text {graviton }}^{2}\right)\right)}
$$

Obviously, the issue of whether or not a graviton has a mass will impact how realistic the approximations given really are, as well as be important to the issue which Leszek M. Sokolowski, Andrzej Staruszkiewicz [16] (2006) raised: "The graviton must have features different from those of the photon and these cannot be predicted from classical general relativity." This will impact strongly upon how to analyze the relationship between wavelength $\lambda_{\text {graviton }}$ and frequency, $f$. Note that Sokolowski, et al. (2006) [16] state that there is a decisive break down of application of MEANING? the formula $E=\hbar \cdot \omega$, which means in order to make sense out of the graviton and gravitational wave connection, one really needs to investigate the space-time constraints in which relic gravitons/gravitational waves arose. Note, it is possible that as much as up to $2 / 3$ rds of the initial relic "matter"-energy initial states used in the construction of the early universe was DM, with no dark energy. What would be beneficial would be to delineate whether or not the graviton/gravitational wave, at its genesis is linkable to DM, to understand the original space time in which the universe evolved in. Note that Bert Janssen, Yolanda Lozano [17] in hep-th/0207199 describe a so called massive/"giant" graviton in terms of study, from the microscopical point of view, via a giant graviton configurations where the gravitons expand into an M2-brane, with the topology of a fuzzy 2-sphere. Fine, but stating that AdS5 $\times$ S5 background is used for embedding will not yield experimental confirmation. So in pursuit of experimental confirmation, it is appropriate to examine whether or not gravitons/GW can tie in with DE and/or DM, which have measurable consequences as far as observational cosmology and astrophysics. 


\section{Linkage of DM to gravitons and gravitational waves?}

Let us state that the object of early universe GW astronomy would be to begin with confirmation of whether or not relic GW were obtainable, and then from there to ascertain is there is linkage which can be made to DM production... Durrer, Massimiliano Rinaldi (2009) [18], state that there would be probably negligible for this case (practically nonexistent) graviton production in cosmological eras after the big bang.. In fact, they state that they investigate the creation of massless particles in a Universe which transits from a radiation-dominated era to any other (via an) expansion law. "We calculate in detail the generation of gravitons during the transition to a matter dominated era. We show that the resulting gravitons generated in the standard radiation/matter transition are negligible" This indicated to the author, Beckwith, that it is appropriate to look at the onset of relic GW/Graviton production. Note also that Ruth Durrer, Massimiliano Rinaldi [18] state furthermore in their conclusions: "a graviton spectrum present at the beginning of the radiation era can become significantly amplified and modified by intermediate, non standard evolution of the universe". This is in part what will be suggested. A non standard evolution protocol which delivers One of the cruder ways of delineating the evolution of GW is the super adiabatic approximation, done for when $k^{2} \ll\left|a^{\prime \prime} / a\right|$ as given by M. Giovannini [19] (page 138) of the form, when $\mu_{k} \equiv a \cdot h_{k}$ is a solution to

$$
\mu_{k}^{\prime \prime}+\left[k^{2}-\frac{a^{\prime \prime}}{a}\right] \mu_{k}=0
$$

Which to first order when $h k^{2} \ll\left|a^{\prime \prime} / a\right|$ leads to a GW solution [19]

$$
{ }_{k}(\tau) \cong A_{k}+B_{K} \cdot \int_{0}^{\tau} \frac{\mathrm{d} x}{a(x)}
$$

This will be contrasted with a very similar evolution equation for gravitons, of the form (i.e. KK gravitons in higher dimensions)

$$
h^{\prime \prime}-\left[4 k^{2}+\frac{m^{2}}{a^{2}(z)}\right] h \equiv 0
$$

One of the most frequently appealed to models of linkage between gravitons, and DM is the so called KK graviton, i.e. as a DM candidate. KK gravitons. Note that usual Randal Sundrum brane theory has a production rate [20] of

$$
\Gamma \sim T^{6} / M_{\text {Planck }}^{2}
$$

as the number of Kaluza Klein gravitons per unit time per unit volume Note that this production rate is for a formula assuming mass for which $T_{*}>M_{X}$, and that we are assuming that the temperature $T \sim T_{*}$. Furthermore, we also are looking at a de facto total production rate of $\mathrm{KK}$ gravitons of the form [20]

$$
\frac{\mathrm{d} n}{\mathrm{~d} t} \sim \frac{T^{6}}{M_{\text {Planck }}^{2}} \cdot(T \cdot R)^{d} \sim T^{4} \cdot\left(\frac{T}{M_{X}}\right)^{2+d}
$$

where $\mathrm{R}$ is the assumed higher dimension "size" and, $\mathrm{d}$ is the number of dimensions above 4 , and typically we obtain $\mathrm{T} \gg 1 / \mathrm{R}$. i.e. we can typically assume tiny higher dimensional "dimensions", very high temperatures, and also a wave length for the result- 
ing KK graviton for a DM candidate looking like [20]

$$
\lambda_{\text {KK-Graviton }} \sim T^{-1}
$$

If KK gravitons have the same wavelength as DM, this will support Jack Ng's [7] treatment of DM. All that needs to put this on firmer ground will be to make a de facto linkage of KK Gravitons, as a DM candidate, and more traditional treatments of gravitons, which would assume a steady drop in temperature from $T \sim T^{*}$, to eventually much lower temperature scales. Note that in a time interval based as proportional to the inverse of the Hubble parameter, we have the total numerical density of KK gravitons (on a brane?) as $n(T) \sim T^{2} M_{\text {Planck }}^{*} \cdot(T / M)^{2+d}$, where $M_{\text {Planck }}^{*} \sim 10^{18} \mathrm{GeV}$ give or take an order of magnitude. This number density $n(T)$ needs to be fully reconciled to $\lambda_{\text {KK-Graxiton }} \sim T^{-1}$ and can be conflated with the dimensionality "radius" value $R \sim 10^{\bar{d}} \cdot 10^{-17}$ centimeters for dimensions above 4 space time GR values, with this value of $\mathrm{R}$ being unmanageable for $\mathrm{d}<2$. V.A. Rubakov, (2009) [21] [22] makes the claim of the KK graviton obeying the general Yukawa style potential

$$
V(r)=-\frac{G_{4}}{r} \cdot\left(1+\frac{\text { const }}{k^{2} r^{2}}\right)
$$

As well as being related to an overall wave functional which can be derived from a line element

$$
\mathrm{d} S^{2} \equiv\left[a^{2}(z) \cdot \eta_{u v}+h_{u v}(x, z)\right] \cdot \mathrm{d} x^{u} \mathrm{~d} x^{v}+\mathrm{dz} z^{2}
$$

With $h^{\prime \prime}-\left[4 k^{2}+\frac{m^{2}}{a^{2}(z)}\right] h \equiv 0$ (suppressing the u,v coefficients). This evolution equation for the KK gravitons is very smilar to work done by Baumann, Daniel, Ichiki, Kiyotomo, Steinhardt, Paul J. Takahashi, Keitaro [23] (2007) with similar assumptions, with the result that KK gravitons are a linear combination of Bessel functions. Note that one has for gravitions [20] [21].

$$
h \equiv h_{m}(z \rightarrow 0)=\text { const } \cdot \sqrt{\frac{m}{k}}
$$

Ruth Gregory, Valery A. Rubakov and Sergei M. Sibiryakov [24] (2000) make the additional claim that for large $\mathrm{z}$ (the higher dimensions get significant) that there are marked oscillatory behaviors, i.e. Rapid oscillations as one goes into the space for branes for massive graviton expansion [21], [24].

$$
h \equiv h_{m}(z \neq 0) \approx \text { const } \cdot \sqrt{a(z)} \cdot \sin \left(\frac{m}{k} \cdot \operatorname{esp}(k z)+\varphi_{m}\right)
$$

This is similar to what Baumann, Daniel, Ichiki, Kiyotomo, Steinhardt, Paul J. Takahashi , Keitaro [23] (2007) for GW, in a relic setting, with the one difference being that the representation for a graviton is in the $\mathrm{z}$ (additional dimension) space, as opposed to what Bauman et al. did for their evolution of GW, with an emphasis upon generation in overall GR space time. Furthermore, the equation given in $h^{\prime \prime}-\left[4 k^{2}+\frac{m^{2}}{a^{2}(z)}\right] h \equiv 0$ for massive graviton evolution as KK gravitons along $\mathrm{dS}$ branes is similar to evolution of GW in more standard cosmology that the author, Beckwith, thinks that the main 
challenge in clarifying this picture will be in defining the relationship of dS geometry, in overall Randall Sundrum brane world to that of standard 4 space. We need though, now to look at whether or not higher dimensions are even relevant to GR itself.

\section{Now the Main Topic: How Would DM Be Influenced by Gravitons, in 4 Dimensions}

We will also discuss the inter relationship of structure of DM, with challenges to Gaussianity. The formula as given by [25], [26]

$$
\delta \equiv-\left[\frac{3}{2} \cdot \Omega_{m} \cdot H^{2}\right]^{-1} \cdot \nabla^{2} \Phi
$$

will be gone into the variation, so alluded to which we will link to a statement about the relative contribution of Gaussianity, via looking at the gravitational potential [26] [27]

$$
\Phi \equiv \Phi_{L}+f_{N L} \cdot\left[\Phi_{L}^{2}-\left\langle\Phi_{L}^{2}\right\rangle\right]+g_{N L} \cdot \Phi_{L}^{3}
$$

Here the expression $f_{N L}=$ variations from Gaussianity, while the statements as to what contributes, or does not contribute will be stated in our $\Phi_{L} \equiv$ presentation. Furthermore, is a linear Gaussian potential, and the overall gravitational potential is altered by inputs from the term, presented, $f_{N L}$. The author discussed inputs into variations from Gaussianity, which were admittedly done from a highly theoretical perspective with Sabino Matarre, [26] on July 10, with his contributions to non Guassianity being constricted to a reported range of $-4<f_{N L}<80$. The author, Beckwith, prefers a narrower range along the lines of $0.5<f_{N L}<20$ for reasons which will be gone into, in the text. Needless to state, though, dealing with what we can and cannot measure, what is ascertained as far as DM, via a density profile variation needs to have it reconciled with DM detection values [26]

$$
\sigma_{\text {DM-dectecion }} \leq 3 \times 10^{-8} \mathrm{pb} \text { (pico barns) }
$$

It is note worthy to note that the question of DM/ KK gravitons, and also the mass of the graviton not only has relevance to whether or not, higher dimensions are necessary/ advisable in space time models, but also may be relevant to if massive gravitons may solve/partly fulfill the DE puzzle. To whit, KK gravitons would have a combined sum of Bessel equations as a wave functional representation. In fact V. A. Rubasov [20] [21] writes that KK graviton representation as, after using the following normalization

$$
\int \frac{\mathrm{d} z}{a(z)} \cdot\left[h_{m}(z) \cdot h_{\tilde{m}}(z)\right] \equiv \delta(m-\tilde{m})
$$

where $J_{1}, J_{2}, N_{1}, N_{2}$ are different forms of Bessel functions, to obtain the KK graviton/DM candidate representation along RS dS brane world [20] [21]

$$
h_{m}(z)=\sqrt{m / k} \cdot \frac{J_{1}(m / k) \cdot N_{2}([m / k] \cdot \exp (k \cdot z))-N_{1}(m / k) \cdot J_{2}([m / k] \cdot \exp (k \cdot z))}{\sqrt{\left[J_{1}(m / k)\right]^{2}+\left[N_{1}(m / k)\right]^{2}}}
$$

This allegedly is for $\mathrm{KK}$ gravitons having an order of $\mathrm{TeV}$ magnitude mass $M_{Z} \sim k$ (i.e. for mass values at $0.5 \mathrm{TeV}$ to above a $\mathrm{TeV}$ in value) on a negative tension $\mathrm{RS}$ brane. What would be useful would be managing to relate this KK graviton, which is moving 
with a speed proportional to $H^{-1}$ with regards to the negative tension brane with $h \equiv h_{m}(z \rightarrow 0)=$ const $\cdot \sqrt{\frac{m}{k}}$ as a possible initial starting value for the KK graviton mass, before the KK graviton, as a "massive" graviton moves with velocity $H^{-1}$ along the RS dS brane. If so, and if $h \equiv h_{m}(z \rightarrow 0)=$ const $\cdot \sqrt{\frac{m}{k}}$ represents an initial state, then one may relate the mass of the KK gravition, moving at high speed, with the initial rest mass of the graviton, which in four space in a rest mass configuration would have a mass many times lower in value, i.e. of at least $m_{\text {graviton }}(4-\operatorname{Dim} G R) \sim 10^{-48} \mathrm{eV}$, as opposed to $M_{X} \sim M_{\mathrm{KK}-\text { Graviton }} \sim .5 \times 10^{9} \mathrm{eV}$. Whatever the range of the graviton mass, it may be a way to make sense of what was presented by Dubovsky, Flauger, Starobinsky, and Thackev [28] (2009) who argue for graviton mass using CMBR measurements, of up to $m_{\text {graviton }}(4-\operatorname{Dim} G R) \sim 10^{-20} \mathrm{eV}$. This can be conflated with Marcio E. S. Alves, Oswaldo D. Miranda, Jose C. N. de Araujo's results arguing that non zero graviton mass may lead to acceleration of our present universe, in a manner usually conflated with $\mathrm{DE}$, i.e. their graviton mass would be about $m_{\text {graviton }}(4-\operatorname{Dim} G R) \sim 10^{-48} \times 10^{-5} \mathrm{eV} \sim 10^{65}$ grams, leading to a possible explanation for when the universe accelerated, i.e. the de-acceleration parameter, due to changes in the scale factor, written as [29] [30] [31]

$$
q=-\frac{\ddot{a} a}{\dot{a}^{2}}
$$

In the case of working with a simpler version of the Friedman equation with no graviton mass, but with pressure and density factored in, we can obtain [29] [30] [31]

$$
\frac{\ddot{a}}{a}=\frac{4 \pi G}{3} \cdot\left[\left(-3 p / c^{2}\right)-\rho\right]
$$

This will lead to a very simple de celebration parameter value of [29] [30] [31]

$$
q=-\frac{\ddot{a} a}{\dot{a}^{2}}=\left(\frac{4 \pi G}{3 c^{2} H^{2}}\right) \cdot[3 p+\rho]
$$

The article will see what happens to insure whether or not the sign of Equation (25) and Equation (26) and Equation (26) goes from positive to negative. Needless to say, if one has a graviton mass $m_{\text {graviton }} \neq 0$, then Equation (25) changes, and there will be a way forward to consider whether or not using a modification of GR, with scale factor evolution of , with non zero graviton mass terms added in to obtain [29] [30] [31]

$$
\left(\frac{\dot{a}}{a}\right)^{2}+\frac{m_{g}^{2} c^{4}}{2 \hbar^{2}} \cdot\left(1-a^{2}\right) \equiv \frac{8 \pi G}{3 c^{2}} \cdot \rho
$$

And [29] [30] [31]

$$
\frac{\ddot{a}}{a}+0.5 \cdot\left(\frac{\dot{a}^{2}}{a^{2}}\right)+\frac{m_{g}^{2} c^{4}}{4 \hbar^{2}} a^{2} \cdot\left(a^{2}-1\right)=\frac{8 \pi G}{3 c^{2}} \cdot p
$$

For the matter dominated era, it is important to note that the R.H.S. of Equation (28) is zero. This leads to Equation (26) having increasingly positive acceleration values as would be definitely be given for masses of $m_{\text {graviton }}(4-\operatorname{Dim} G R) \sim 10^{-48} \times 10^{-5} \mathrm{eV} \sim 10^{65}$ grams for red shift values $z \sim 0.3$ for (1.4) just becoming $>0$ to maximum values of 
(1.4) today, with $z=0$, all at mass of the order of $10^{65}$ grams. This increase of Equation (24) then leads us to consider how to configure Equation (27) and Equation (28) and for RS brane world values. There are terms which are added to the first Friedman equation. i.e. when using ultra low graviton mass, where $r_{c}=\frac{M_{P}^{2}}{2 M_{(5)}^{3}}$ and, often $\varepsilon=1$ and $r_{c}$ is usually though of as the separation between branes. i.e. if $r_{c} \rightarrow \infty$, we recover the usual first Friedman equation. For now we write the first Friedman equation for a brane system as [32].

$$
\frac{\dot{a}}{a} \equiv H=\frac{\epsilon}{2 r_{c}}+\sqrt{\frac{8 \pi G \rho}{3}+\frac{1}{4 r_{c}^{2}}}
$$

As can be related to, if we wish to look at string theory versions of the FRW equation, in Friedman-Roberson-Walker metric space, we can do the following decomposition, with different limiting values of the mass, and other expressions, e.g. as a function of an existing cosmological constant.

$$
\left(\frac{\dot{a}}{a}\right)^{2}=\frac{\rho_{\text {Total }}}{3 M_{\text {Planck }}^{2}}-\frac{k}{a^{2}}+\frac{\Lambda}{3}
$$

As well as

$$
\left(\frac{\ddot{a}}{a}\right)=-\frac{\left(\rho_{\text {Total }}+3 p_{\text {Total }}\right)}{6 M_{\text {Planck }}^{2}}+\frac{\Lambda}{3}
$$

Not only this, if looking at the brane theory Friedman equations as presented by/for Randall Sundrum theory, it would be prudent working with [32]

$$
\dot{a}^{2}=\left[\left(\frac{\rho}{3 M_{4}^{2}}+\frac{\Lambda_{4}}{3}+\frac{\rho^{2}}{36 M_{\text {Planck }}^{2}}\right) a^{2}-\kappa+\frac{C}{a^{2}}\right]
$$

For the purpose of Randal Sundrum brane worlds, Equation (32) is what will be differentiated with respect to $\mathrm{d} / \mathrm{d} \tau$, and then terms from (1.5) will be used, and put into a derivable equation which will be for a RS brane world version of $q=-\frac{\ddot{a} a}{\dot{a}^{2}}$. Several different versions of what $\mathrm{q}$ should be will be offered as far as what the time dependence of terms in 1.10 actually is. Note that Roy Maartens has written as of 2004 that KK modes [32] (graviton) satisfy a 4 Dimensional Klein-Gordon equation, with an effective 4 dim mass, $m_{n}($ Graviton $)=\frac{n}{L}$, with $m_{0}($ Graviton $)=0$, and $\mathrm{L}$ as the stated "dimensional value" of higher dimensions. The value $m_{0}$ (Graviton) $10^{-65}-10^{-60}$ gram in value picked is very small, but ALMOST zero. Grossing [33] has shown how the Schrodinger and Klein Gordon equations can be derived from classical lagrangians, i.e. using a version of the relativistic Hamilton-Jacobi-Bohm equation, with a wave functional $\psi \sim \exp (-i S / \hbar)$, with $S$ the action, so as to obtain working values of for a tier of purported masses of a graviton from the equation, for $4 \mathrm{D}$ of

$$
\left[g^{\alpha \beta} \partial_{\alpha} \partial_{\beta} \underset{\text { FLAT-SPACE }}{\longrightarrow} \nabla^{2}-\partial_{\tau}^{2}\right]
$$

and 


$$
\left[\nabla^{2}-\partial_{\tau}^{2}\right] \cdot \psi_{n}=m_{n}^{2}(\text { graviton }) \cdot \psi_{n}
$$

If one is adding, instead the small mass of [34]

$$
m_{n}(\text { Graviton })=\frac{n}{L}+10^{-65} \text { rams }
$$

with $m_{0}$ (Graviton $) \approx 10^{-65}$ grams, then the problem being worked with is a source term problem of the form given by Peskins [35] as of the type

$\psi_{n}(x) \equiv \int \mathrm{d}^{3} p \cdot \frac{1}{(2 \pi)^{3}} \cdot \frac{1}{\sqrt{2 E_{P}}} \cdot\left\{\left(a_{P}+\frac{i}{\sqrt{2 E_{P}}} \cdot F T\left(m_{0}(\right.\right.\right.$ graviton $\left.\left.)\right)\right) \exp (-i p x)+$ H.C. $\}$

This is, using the language V.A. Rubakov [20] [21] put up equivalent to writing, using Equation (28)

$\psi_{m}(x) \approx h_{m}(x)+\int \mathrm{d}^{3} p \cdot \frac{1}{(2 \pi)^{3}} \cdot\left(\frac{1}{\sqrt{2 E_{P}}}\right)^{2} \cdot\left\{\left(i \cdot F T\left(m_{0}(\right.\right.\right.$ graviton $\left.\left.\left.)\right)\right) \exp (-i p x)+H . C.\right\}$

i.e. how to inteprept the quantity $F T\left(m_{0}\right.$ (graviton)) being the issue which will be covered in this document. If $m_{0}$ (graviton) is a constant, then the expression Equation (37) has delta functions. This goes into evaluating, then, momentum, appropriately. We will do a time differentiation of Equation (35) in this document, and compare it term by term with what arises if there is a sutiable graviton mass, and comment as to what would be needed to have graviton mass in a brane version of Equation (32), and its time derivative, and do a similar analysis as to what was done to recover the positive acceleration, for Equation (29) using brane equivalents to Equation (30) as well as imputs from Equation (31). Now why is this important? This datum may especially show up about modification of the typical galaxy models, as follows

A. Not everyone buys dark energy. i.e. Controversies of DM/DE applications to cosmology. How HFGW may help resolve them

The following is meant as a travelogue as to current problems in cosmology which will require significant revision of our models. Exhibit $\mathrm{A}$ as to what to consider is the cosmic void hypothesis'. See Timothy Clifton, Pedro G. Ferreira and Kate Land [36]. i.e. Clifton raises the following question-can HFGW and detectors permit cosmologist to get to the bottom of this? "Solving Einstein's equations for an averaged matter distribution is NOT the same as solving for the real matter distribution and then averaging the resultant geometry" ("We average, then solve when in effect we should solve, then average").

Next, let us look at a recently emerging conundrum of DM feeding into the structure of new galaxies and their far earlier than expected development, i.e. 5 billion years after the big bang. Galaxy formation issues... Hierarchical Galaxy Formation theory at a glance usually proceeds as follows. i.e. what happens when the following diagram of simple addition of new structure no longer holds ? This is very significant, since when the significant formation of galaxies occurs, as of about $z \sim 0.2$ is before the turn up in the expansion rate for the universe, which will be referenced as of occurring about $z \sim 0.5-0.55$. What do we do if, as an example, find that the initial start of galaxy formation occurred five billion years ago, at, say $Z \sim 0.5$. What could cause the earlier clumping? 
Several scenarios which will be investigated. First of all, note the formula of variation of DM density which exists has, among other things a Hubble parameter $H$, and also the $2^{\text {nd }}$ derivative of the gravitational potential $\nabla^{2} \Phi$, where $\rho_{0}, a_{0}$ are today's values for density and "distance". Note that if the following happen

$$
\begin{aligned}
H^{2}=\left(\frac{\dot{a}}{a}\right)^{2}= & {\left[\left(\frac{\rho}{3 M_{4}^{2}}+\frac{\Lambda_{4}}{3}+\frac{\rho^{2}}{36 M_{\text {Planck }}^{2}}\right)-\frac{\kappa}{a^{2}}+\frac{C}{a^{4}}\right] \underset{\substack{\kappa \rightarrow 0, \Lambda_{4} \rightarrow 0}}{\longrightarrow}\left[\frac{\rho}{3 M_{4}^{2}}+\frac{\rho^{2}}{36 M_{\text {Planck }}^{2}}+\frac{C}{a^{4}}\right] } \\
& \rho \rightarrow \rho(z) \equiv \rho_{0} \cdot(1+z)^{3}-\left[\frac{m_{g}}{8 \pi G}\right] \cdot\left(\frac{a_{0}^{4}}{14 \cdot(1+z)^{4}}+\frac{2 a_{0}^{2}}{5 \cdot(1+z)^{2}}-\frac{1}{2}\right)
\end{aligned}
$$

and

$$
1+z=a_{0} / a
$$

then the contribution of large $Z$, i.e. large contributions from red shift, that a significant early contributions will be for non zero contributions from $1 / \rho^{\beta}$ terms, for [large number] $>\beta \geq 1$ in the $\mathrm{DM}$ density variation parameters. So long as $m_{\text {graviton }} \neq 0$, even if $m_{\text {graviton }}$ is very small. In addition, if the following is true, Equation (20), then there are contributions from terms to be considered.

When using the formula, $\nabla^{2} \Phi$ consider the contributions to the expression $f_{N L}$. To do this consider first what [37] Licia Verde (2000) put up about $\Phi$ considered to be the gravitational potential, and $\Phi_{L}$ its linear Gaussian contribution. This has been improved upon by, recently, P. Chingabam, C. Park (2009) [38] improved upon the simulation done by Verde [37] (2003), who worked with $f_{N L}$ bounded as follows: $10^{-4}<f_{N L}<10^{-2}$, whereas the Chingabam, Park (2009) [38] publication considered $-4<f_{N L}<80$ at a confidence level of $95 \%$. One of the simpler suppositions a person could use is what would be involved if,

$$
\lambda_{\text {Graviton }}=\left(h / m_{\text {graviton }} \cdot c\right) \equiv 1 / m_{\text {graviton }}
$$

Then

$$
\left(1+\frac{f_{N L} \cdot\left[\Phi_{L}^{2}-\left\langle\Phi_{L}\right\rangle^{2}\right]}{\Phi_{L}}\right) \propto\left[1-\frac{r}{\lambda_{\text {graviton }}}\right] \sim \exp \left(-r / \lambda_{\text {graviton }}\right)
$$

Alternately, if the brane theory model of a gravitational potential were used, with KK graviton modes, then

$$
\left(1+\frac{f_{N L} \cdot\left[\Phi_{L}^{2}-\left\langle\Phi_{L}\right\rangle^{2}\right]}{\Phi_{L}}\right) \propto\left[1+\frac{\text { const }}{r^{2} k^{2}}\right]
$$

Now for some sort of bounds as to what may be acceptable bounds in error, based upon CMB data

$$
\left|f_{N L} \cdot\left[\Phi_{L}^{2}-\left\langle\Phi_{L}^{2}\right\rangle\right]\right| \leq 10^{-5} \cdot\left|f_{N L}\right|<\text { ? up to } 10^{-3}
$$

Depending upon which model is used for describing $\Phi_{L}$ i.e. as a perturbation of a gravitational potential, this Equation (44) may allow us to obtain a good guess as to what dimensions are crucial for the formation of a graviton, i.e. how much spread may 
be permitted. Note that $\Phi_{L}$, is a linear approximation to the gravitational potential given . In addition, one can, as a crude approximation write to first order $\Phi_{L} \sim 1 / r$. Also the parameter $f_{N L}$ is usually, often with partly sinusoidal variation, taken from primordial non gaussianity traces taken from the CMBR itself.

Non-linear dynamics at recombination will lead to, for CMBR a treatment similar to what was given by Jean-Luc Lehners, Paul J. Steinhardt, (2009) [39], i.e., if $\tilde{\Phi}_{H}$ is the Bardeen Space-Space metric perturbation, and $\tilde{\Phi}_{L}$ a Bardeen Space-Space perturbation to linear order. Here, Bardeen Space-Space metric perturbation is defined by James M. Bardeen (1982) [40]

$$
\tilde{\Phi}_{H}=\tilde{\Phi}_{L}+f_{N L} \cdot \tilde{\Phi}_{L}^{2}
$$

Also, White and $\mathrm{Hu}$ [41] (1996), also have a convenient way to link the gravitational potential $\Phi$ to temperature fluctuations, and do it as, when assuming $\Phi_{\text {final }}$ is ignorable

$$
\left.\frac{\Delta T}{T}\right|_{\text {Final }}-\left.\frac{\Delta T}{T}\right|_{\text {Initial }}=-\Phi_{\text {Initial }}
$$

A simple way to understand what is said by Equation (46) is to consider if or not it is linkable to the Sach-Wolfe effect. Here, the Sachs-Wolfe effect (ISW) occurs when the Universe is dominated in its density by something other than matter. If the Universe is dominated by matter, then large-scale gravitational potential wells and hills do not evolve significantly. If the Universe is dominated by radiation, or by dark energy, though, those potentials do evolve, subtly changing the energy of photons passing through them. If there is a major difference in the initial and final ratios $\Delta T / T$ of temperature variations are for different red shift values, and for the Friedman model, to good approximation, $\frac{\delta T}{T}=\phi / 3$, if $c=1$. If the approximations for the Friedman eqn. are valid, then one has, say

$$
(\delta T / T) \cong(1 / 3) \cdot\left[\Phi_{L}+f_{N L} \cdot\left(\Phi_{L}^{2}-\left\langle\Phi_{L}\right\rangle^{2}\right)\right]
$$

Equation (47) has its counterpart in what Daniel Babich, Paolo Creminelli, Matias Zaldarriaga [42] (2004) about the influence of curvature "perturbations" with some of them being linear, and the others showing a slight perturbative effect. i.e. look at

$$
\varsigma(x) \equiv \varsigma_{\text {Gaussian }}(x)-\frac{3}{5} f_{N L} \cdot\left[\varsigma_{\text {Gaussian }}^{2}(x)-\left\langle\varsigma_{\text {Gaussian }}(x)\right\rangle^{2}\right]
$$

Here, $\varsigma_{\text {Gaussian }}(x)$ is with regards to a Gaussian perturbation of curvature. Where, $\varsigma_{\text {Gaussian }}(x)$, is as defined by David H. Lyth [43] (2005)

$$
\varsigma_{\text {Non-Gaussian }}(x) \equiv \sum_{i} N_{i} \delta \phi_{i}+\frac{1}{2} \cdot \sum_{i j} N_{i j} \delta \phi_{i} \delta \phi_{j} \underset{\text { Single-field }}{\longrightarrow} N_{\sigma} \delta \sigma+N_{\sigma \sigma}(\delta \sigma)^{2}
$$

where we use

$$
N_{\sigma} \equiv \partial N / \partial \sigma, N_{\sigma \sigma} \equiv \partial^{2} N / \partial^{2} \sigma, \text { where } N=\int H \mathrm{~d} \tau
$$

What is actually observed, contradicts this halo emerging history "tree", i.e. Although this "story" for DM seems to be well established. i.e. Just ONE little problem: DM appears to be fattening up young galaxies, allowing for far-earlier-than-expected creation 
of early galaxies. "A clutch of massive galaxies that seem to be almost fully-formed just 5 billion years after the big bang challenge models that suggest galaxies can only form slowly. Tendrils of dark matter that fed the young galaxies on gas could be to blame (NASA/CXC/ESO/P Rosati et al.)" [45] leading to [46].

Needless, to say though, an analysis of the influence of DM on structure formation would have to take into consideration the datum presented by [47] as reporting upon the data analysis of G. Hinsaw [48] and others as to the relative super abundance of DM in early universe conditions. i.e. considering the following. The relative imprecision of graviton measurement, can be given as follows. This is a measurement in particle physics, and if the KK graviton is linkable to DM, it means that we will have to have very good ways to test for production rates, as will be argued later. The following below is a typical representation of the KK tower model for gravitons, with the zeroth KK mode being approximately the 4 dimensional graviton. From scattering data, the relative mass contributions show up, as follows for KK gravitons, as modeled below. These representation are for leading up to investigating if or not one needs KK gravitons, as either a semi classical, or a brane theory/string theory construct, as a was of determining if either.

1) Gravitons have mass, i.e. $m_{\text {graviton }} \sim 10^{-65}$ grams $\sim 10^{-48}$ electron volts

2) DE style expansion at $Z \sim 0.55$ can be seen as a consequences of 1) above. Does this necessitate or imply that KK gravitons should be presented as a quantum gravity theory?

3) DM may be connected with KK variants of higher dimensional generalizations of $m_{\text {graviton }}$ mass

4) The issue of if or not gravitons/entropy/constituent DM may be linked to instanton physics models of gravitons Understanding the KK gravitons as a DM candidate may permit us to understand how DM and DE are inter related. See as given below. The discussion of such will involve coherent state of gravitons as contributors to GW. This is the message of Figure 2 below.
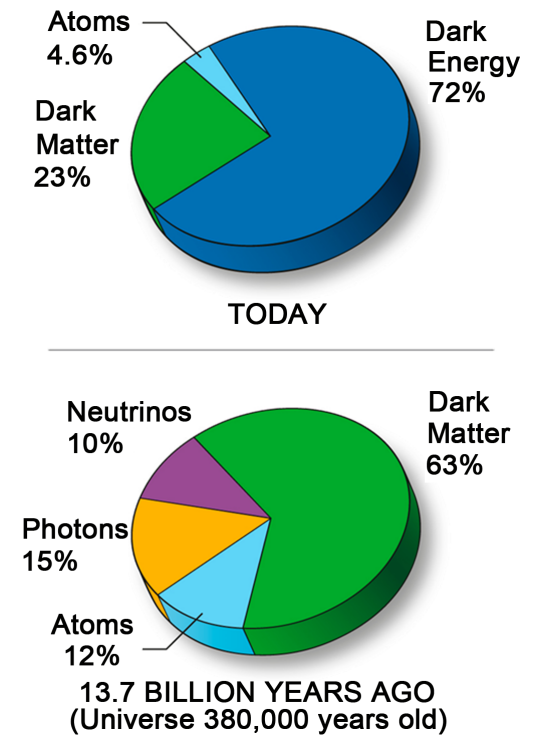

Figure 2. From G. Hingsaw presentation, in COMO, Italy, July 2009 at the ISAPP [49]. 
How DM, and other constituent parts of the early 380 thousand year old universe evolved to have connections with KK gravitons is connected closely with the following

\section{B. Issues about Coherent state of Gravitons (linking gravitons with GW)}

In the quantum theory of light (quantum electrodynamics) and other bosonic quantum field theories, coherent states were introduced by the work of Roy J. Glauber in 1963 [50] Now, it is well appreciated that Gravitons are NOT similar to light. So what is appropriate for presenting gravitons as coherent states? Coherent states, to first approximation are retrievable as minimum uncertainty states. If one takes string theory as a reference, the minimum value of uncertainty becomes part of a minimum uncertainty which can be written as given by Maggiorie, [51] and Venziano [52], where $l_{S} \cong 10^{\alpha} \cdot l_{\text {Planck }}$, with $\alpha>0$, and $l_{\text {Planck }} \approx 10^{-33}$ centimeters

$$
\Delta x>\frac{\hbar}{\Delta p}+\frac{l_{S}^{2}}{\hbar} \cdot[\Delta p]
$$

To put it mildly, if we are looking at a solution to minimize graviton position uncertainty, we will likely be out of luck if string theory is the only tool we have for early universe conditions. Mainly, the momentum will not be small, and uncertainty in momentum will not be small either. Either way, most likely, $\Delta x>l_{S} \cong 10^{\alpha} \cdot l_{\text {Planck }}$ In addition, it is likely, as Klaus Kieffer in the book "Quantum Gravity" [53] on page 290 of that book that if gravitons are excitations of closed strings, then one will have to look for conditions for which a coherent state of gravitons, as stated by Mohaupt (2003) [54] occurs. What Mohaupt is referring to is a string theory way to re produce what Ford gave in 1995 [55] i.e. conditions for how Gravitons in a squeezed vacuum state, the natural result of quantum creation in the early universe will introduce metric fluctuations. Ford's [55] (1995) treatment is to have a metric averaged retarded Green's function for a massless field becoming a Gaussian. The condition of Gaussianity is how to obtain semi classical, minimal uncertainty wave states, in this case de rigor for coherent wave function states to form. Ford uses gravitons in a so called "squeezed vacuum state" as a natural template for relic gravitons. i.e. the squeezed vacuum state (a squeezed coherent state) is any state such that the uncertainty principle is saturated: In QM coherence would be when $\Delta x \Delta p=\hbar / 2$. In the case of string theory it would have to be $\Delta x \Delta p=\frac{\hbar}{2}+\frac{l_{S}^{2}}{2 \cdot \hbar} \cdot[\Delta p]^{2}$. Putting it mildly, the string theory case is far more difficult. And that is the problem, with regards to string theory, what is an appropriate vacuum expectation value for treating a template of how to nucleate gravitons into a coherent state with respect to relic conditions. Ford, in 1994 [55], wrote a squeezed state operation $S()$ via $|\varsigma\rangle=S(\varsigma) \cdot|0\rangle$, Here, the operator. $|0\rangle$ is a ground state, and frequently, as Ford did, in 1994 [55], there is a definition of a root mean squared fluctuation of a graviton/gravitational wave state via use of an average scalar field $\Phi$, where

$$
h^{2}=\frac{1}{30} \cdot\left\langle h_{i j} h^{i j}\right\rangle=\left.\frac{1}{15} \cdot\left\langle\Phi^{2}\right\rangle \equiv \frac{1}{180} \cdot T^{2}\right|_{\text {Thermai-bath }}
$$

Here, the value $\left.T\right|_{\text {Thermai-bath }}$ has yet to be specified, and that actually for energy values approximately of the order of $10^{15} \mathrm{GeV}$ which may be the mean temperature for the expanding universe mid way, to the end of inflation, which does not equal current even smaller string theory estimates as presented by Li et al. 
$\left.h\right|_{\text {THERMAL-BATH }} \approx 10^{-18} / \sqrt{\mathrm{Hz}} \neq h_{\text {rms }} \sim 10^{-30}-10^{-34} / \sqrt{\mathrm{Hz}} \equiv$ string theory values for inflationary Gravitational amplitudes. i.e. the more modern treatments are predicting almost infinitesimal GW fluctuations. It is not clear from Ford's 1995 treatment of gravitons, and fluctuations, if he is visualizing fluctuation of gravitons/GW, but if one takes literally (1.14) as a base line, and then considering what would be the optimal way to obtain a way to obtain coherent states of gravitons, going to the $\mathrm{Li}$ [5] stated value of $h_{r m s} \sim 10^{-39} / \sqrt{\mathrm{Hz}}$ for solar plasma from the sun as a graviton source, would be a way of obtaining fluctuations $10^{-5}-10^{-9}$ times weaker, i.e. going to $h_{r m s}$ values so small that the requirement for a minimum fluctuation, in line with not contradicting $\Delta x \Delta p=\frac{\hbar}{2}+\frac{l_{S}^{2}}{2 \cdot \hbar} \cdot[\Delta p]^{2}$, if we consider experimental conditions for obtaining $\Delta x \approx h_{r m s} \sim 10^{-39} / \sqrt{\mathrm{Hz}}$. Note that this would put severe restrictions upon the variations in momentum. A subject which will be referenced in whether or not the Li-Baker detector can suitably obtain such small values of $\Delta x \approx h_{\text {rms }} \sim 10^{-39} / \sqrt{\mathrm{Hz}}$ in detection capacity. To do so will require an investigation into extreme sensitivity requirements, for this very low value of $h_{r m s}$. Fanguy Li. et al. (2008) [5] reports in their PRD document $h_{r m s} \sim 10^{-26}-10^{-30} / \sqrt{\mathrm{Hz}}$ would require up to $10^{5}$ seconds in evaluative time for a clean signal, for GW. What will be asked in further sections is if or not the $10^{5}$ seconds in evaluative time for a clean signal can evaluate additional data. i.e. what if one would have to do to distinguish if or not coherent states of gravitons which merge to form GW may be measured via the protocols brought up by Li et al. (2008) for relic GW?

C. Can any detector measure $h_{r m s} \sim 10^{-39} / \sqrt{\mathrm{Hz}}$ ? How squeezed state conditions at the onset of inflation affects usual attempts at measurement of coherent relic graviton states.

Not now. Current limits would be, for $h_{r m s} \sim 10^{-32} / \sqrt{\mathrm{Hz}}$ as a de facto limit for sensitivity. Now what could be said about forming states close to classical representations of gravitons? Venkatartnam, and Suresh, 2007 [56] built up a coherent state via use of a displacement operator $D(\alpha) \equiv \exp \left(\alpha \cdot a^{+}-\alpha^{*} \cdot a\right)$, applied to a vacuum state , where $\alpha$ is a complex number, and $a, a^{+}$as annihilation, and creation operations $\left[a, a^{+}\right]=1$, where one has

$$
|\alpha\rangle=D(\alpha) \cdot|0\rangle
$$

However, what one sees in string theory, is a situation where a vacuum state as a template for graviton nucleation is built out of an initial vacuum state, $|0\rangle$. To do this though, as Venkatartnam, and Suresh did [56], involved using a squeezing operator $Z[r, \vartheta]$ defining via use of a squeezing parameter $r$ as a strength of squeezing interaction term, with $0 \leq r \leq \infty$, and also an angle of squeezing, $-\pi \leq \vartheta \leq \pi$ as used in

$$
Z[r, \vartheta]=\exp \left[\frac{r}{2} \cdot\left([\exp (-i \vartheta)] \cdot a^{2}-[\exp (i \vartheta)] \cdot a^{+2}\right)\right]
$$

where combining the $Z[r, \vartheta]$ with Equation (53) leads to a single mode squeezed coherent state, as they define it via

$$
|\varsigma\rangle=Z[r, \vartheta]|\alpha\rangle=Z[r, \vartheta] D(\alpha) \cdot|0\rangle \underset{\alpha \rightarrow 0}{\longrightarrow} Z[r, \vartheta] \cdot|0\rangle
$$

The right hand side of Equation (54) given above becomes a highly non classical operator, i.e. in the limit that the super position of states $|\varsigma\rangle \underset{\alpha \rightarrow 0}{\longrightarrow} Z[r, \vartheta] \cdot|0\rangle$ occurs, 
there is a many particle version of a "vacuum state" which has highly non classical properties. Squeezed states, for what it is worth, are thought to occur at the onset of vacuum nucleation, but what is noted for $|\varsigma\rangle \underset{\alpha \rightarrow 0}{\longrightarrow} Z[r, \vartheta] \cdot|0\rangle$ being a super position of vacuum states, means that classical analog is extremely difficult to recover in the case of squeezing, and general non classical behavior of squeezed states. Can one, in any case, faced with $|\alpha\rangle=D(\alpha) \cdot|0\rangle \neq Z[r, \vartheta] \cdot|0\rangle$ do a better job of constructing coherent graviton states, in relic conditions, which may not involve squeezing? Note L. Grishchuk wrote in (1989) [57] in "On the quantum state of relic gravitons", where he claimed in his abstract that 'It is shown that relic gravitons created from zero-point quantum fluctuations in the course of cosmological expansion should now exist in the squeezed quantum state. The authors have determined the parameters of the squeezed state generated in a simple cosmological model which includes a stage of inflationary expansion. It is pointed out that, in principle, these parameters can be measured experimentally'. Grishchuk, et al., [58] reference their version of a cosmological perturbation $h_{\mathrm{nlm}}$ via the following argument. How we work with the argument will affect what is said about the necessity, or lack of, of squeezed states in early universe cosmology. From Class. Quantum Gravity: 6 (1989), L 161-L165 [58], where $h_{\mathrm{nlm}}$ has a component $\mu_{\mathrm{nlm}}(\eta)$ obeying a parametric oscillator equation, where $\mathrm{K}$ is a measure of curvature which is $= \pm 1,0, a(\eta)$ is a scale factor of a FRW metric, and $n=2 \pi \cdot[a(\eta) / \lambda]$ is a way to scale a wavelength, $\lambda$, with $\mathrm{n}$, and with $a(\eta)$

$$
\begin{gathered}
h_{\mathrm{nlm}} \equiv \frac{l_{\text {Planck }}}{a(\eta)} \cdot \mu_{\mathrm{nlm}}(\eta) \cdot G_{\mathrm{nlm}}(x) \\
\mu_{\mathrm{nlm}}^{\prime \prime}(\eta)+\left(n^{2}-K-\frac{a^{\prime \prime}}{a}\right) \cdot \mu_{\mathrm{nlm}}(\eta) \equiv 0
\end{gathered}
$$

If $y(\eta)=\frac{\mu(\eta)}{a(\eta)}$ is picked, and a Schrodinger equation is made out of the Lagrangian used to formulate Equation (56) above, with $\hat{P}_{y}=\frac{-i}{\partial y}$, and $M=a^{3}(\eta)$, $\Omega=\frac{\sqrt{n^{2}-K^{2}}}{a(\eta)}, \quad \breve{a}=\left[a(\eta) / l_{\text {Planck }}\right] \cdot \sigma$, and $F(\eta)$ an arbitrary function. $y^{\prime}=\partial y / \partial \eta$. Also, we have a finite volume $V_{\text {finite }}=\int \sqrt{{ }^{(3)} g} \mathrm{~d}^{3} x$

Then the Lagrangian for deriving Equation (56) is (and leads to a Hamiltonian which can be also derived from the Wheeler De Witt equation), with $\varsigma=1$ for zero point subtraction of energy

$$
\begin{gathered}
L=\frac{M \cdot y^{\prime 2}}{2 a(\eta)}-\frac{M^{2} \cdot \Omega^{2} a \cdot y^{2}}{2}+a \cdot F(\eta) \\
\frac{-1}{i} \cdot \frac{\partial \psi}{a \cdot \partial \eta} \equiv \hat{H} \psi \equiv\left[\frac{\hat{P}_{y}^{2}}{2 M}+\frac{1}{2} \cdot M \Omega^{2} \hat{y}^{2}-\frac{1}{2} \cdot \varsigma \cdot \Omega\right] \cdot \psi
\end{gathered}
$$

then there are two possible solutions to the S.E. Grushchuk created in 1989 [57], one a non squeezed state, and another a squeezed state. So in general we work with 


$$
y(\eta)=\frac{\mu(\eta)}{a(\eta)} \equiv C(\eta) \cdot \exp (-B \cdot y)
$$

The non squeezed state has a parameter $\left.B\right|_{\eta} \underset{\eta \rightarrow \eta_{b}}{\longrightarrow} B\left(\eta_{b}\right) \equiv \omega_{b} / 2$ where $\eta_{b}$ is an initial time, for which the Hamiltonian given in Equation (58) in terms of raising/lowering operators is "diagnonal", and then the rest of the time for $\eta \neq \eta_{b}$, the squeezed state for $y(\eta)$ is given via a parameter B for squeezing which when looking at a squeeze parameter $\mathrm{r}$, for which $0 \leq r \leq \infty$, then Equation (59) (1.21) has, instead of $B\left(\eta_{b}\right) \equiv \omega_{b} / 2$

$$
\left.B\right|_{\eta} \underset{\eta \neq \eta_{b}}{\longrightarrow} B\left(\omega, \eta \neq \eta_{b}\right) \equiv \frac{i}{2} \cdot \frac{(\mu / a(\eta))^{\prime}}{(\mu / a(\eta))} \equiv \frac{\omega}{2} \cdot \frac{\cosh r+[\exp (2 i \vartheta)] \cdot \sinh r}{\cosh r-[\exp (2 i \vartheta)] \cdot \sinh r}
$$

Taking Grishchuck's formalism literally, a state for a graviton/GW is not affected by squeezing when we are looking at an initial frequency, so that $\omega \equiv \omega_{b}$ initially corresponds to a non squeezed state which may have coherence, but then right afterwards, if $\omega \neq \omega_{b}$ which appears to occur whenever the time evolution,

$\eta \neq \eta_{b} \Rightarrow \omega \neq \omega_{b} \Rightarrow B\left(\omega, \eta \neq \eta_{b}\right) \equiv \frac{i}{2} \cdot \frac{(\mu / a(\eta))^{\prime}}{(\mu / a(\eta))} \neq \frac{\omega_{b}}{2} \quad$ A reasonable research task would be to determine, whether or not $B\left(\omega, \eta \neq \eta_{b}\right) \neq \frac{\omega_{b}}{2}$ would correspond to a vacuum state being initially formed right after the point of nucleation, with $\omega \equiv \omega_{b}$ at time $\eta \equiv \eta_{b}$ with an initial cosmological time some order of magnitude of a Planck interval of time $t \approx t_{\text {Planck }} \propto 10^{-44}$ seconds The next section will be to answer whether or not there could be a point of no squeezing, as Grishchuck implied [57], for initial times, and initial frequencies, and an immediate transition to times, and frequencies afterwards, where squeezing was mandatory. Note that in 1993 [58], Grischchuk further extended his analysis, with respect to the same point of departure, i.e. What to do with when $|\alpha\rangle=D(\alpha) \cdot|0\rangle \neq Z[r, \vartheta] \cdot|0\rangle$. Having $|\alpha\rangle=D(\alpha) \cdot|0\rangle$ with $D(\alpha)$ a possible displacement operator, seems to be in common with $B\left(\eta_{b}\right) \equiv \omega_{b} / 2$, whereas $|\alpha\rangle=Z[r, \vartheta] \cdot|0\rangle$ which is highly non classical seems to be in common with a solution for which $B\left(\omega_{b}\right) \neq\left(\omega_{b} / 2\right)$ This leads us to the next section, i.e. does $B\left(\eta_{b}\right) \equiv \omega_{b} / 2$ when of time $t \approx t_{\text {Planck }} \propto 10^{-44}$ seconds, and then what are the initial conditions for forming "frequency" $\omega \equiv \omega_{b}$ ? The reader is urged to read [59] and [60] which gives further dimensions to his thinking.

\section{Necessary and sufficient conditions for String/Brane theory graviton cohe-} rent states?

A curved spacetime is a coherent background of gravitons, and therefore in string theory is a coherent state Joseph Gerard Polchinski [3] starting with the typical small deviation from flat space times as can be written up by $G_{u v}(X)=\eta_{u v}+h_{u v}(X)$, with $\eta_{u v}$ flat space time, and the Polyakov action, is generalized as follows, the $S_{\sigma}$ Polyakov action is computed and compared with exponentiated values

$$
S_{\sigma}=\frac{1}{4 \pi \alpha^{\prime}} \cdot \int_{M} \mathrm{~d}^{2} \sigma \cdot \sqrt{g} \cdot g^{a b} \cdot G_{u v}(X) \cdot \partial_{a} X^{\mu} \partial_{b} X^{v}
$$


becomes [3]

$$
\exp \left(-S_{\sigma}\right)=\left[\exp \left(-S_{P}\right)\right] \cdot\left[1-\frac{1}{4 \pi \alpha^{\prime}} \cdot \int_{M} \mathrm{~d}^{2} \sigma \cdot \sqrt{g} \cdot g^{a b} \cdot h_{u v}(X) \cdot \partial_{a} X^{\mu} \partial_{b} X^{v}+\cdots\right]
$$

Polochinski [3] writes that the term of order $h$ in Equation (62) is the vertex operator for the graviton state of the string, with $h_{u v}(X) \equiv-4 \pi g_{c} \cdot \exp \left[-i k X_{S_{u v}}\right]$, and the action of $S_{\sigma}$ a coherent state of a graviton. Now the important question to ask, is if this coherent state of a graviton, as mentioned by Polochinski [3] can hold up in relic, early universe conditions. Rainer Dick [61], in 2001, argued as stating that the "graviton multiplet as one particular dark matter source in heterotic string theory. In particular, it is pointed out that an appreciable fraction of dark matter from the graviton multiplet requires a mass generating phase transition around $T_{C}=10^{8} \mathrm{Gev}$, where the symmetry partners of the graviton would evolve from an ultrahard fluid to pressureless dark matter. Indicates $m \simeq 10 \mathrm{Mev}$ for the massive components of the graviton multiplet". This has a counter part in a presentation made by Berkenstein [62] (2004) with regards to BPS states, and SHO models for $\operatorname{AdS}_{5} \times S^{5}$ geometry. The upsot is that string theory appears to construct coherent graviton states, but it has no answer to the problem that Ford [55] (1995), Grishchuck [57] [58], wrote on if the existing graviton coherent states would be squeezed into non classical configurations in relic conditions.

E. Does LQG give us more direct arguments as to coherent states, squeezed states, and the breakdown of classical behavior at the onset of inflation?

Carlo Rovelli [63], in 2006, in a PRL article states that a vertex amplitude that contributes to a coherent graviton state is the exponential of the Regge action: the other terms, that have raised doubts on the physical viability of the model, are suppressed by the phase of the vacuum state, and Rovelli writes a coherent vacuum state as given by a Gaussian peaked on parts of the boundary $\Sigma_{d}$ of a four dimensional sphere.

$$
\Psi_{q}[s]=\Psi_{q}\left(\Gamma, j_{m, n}\right)
$$

Rovelli states that "bad" contributions to the behavior of Equation (63) are cancelled out by an appropriate (Gaussian?) vacuum wave functional which has "appropriately" chosen contributions from the boundary $\Sigma_{d}$ of a four dimensional sphere. This is to avoid trouble with "bad terms" from what is known as the Barret-Crane vertex amplitude contributions, which are can be iminized by an appropriate choice of vacuum state amplitude being picked. Rovelli [63] calculated some components of the graviton twopoint function and found that the Barrett-Crane vertex yields a wrong long-distance limit. A problem, as stated by Lubos Motel [64] (2007), that there are infinitely many other components of the correlators in the LQG that are guaranteed not to work unless an infinite number of adjustments are made. The criticism is harsh, but until one really knows admissible early universe geometry one cannot rule out the Rovelli [63] approach, or confirm it. In addition, Jakub Mielczarek [65] (2009) considered tensor perturbations produced at a bounce phase in presence of the holonomy corrections. Here bounce phase and holonomy corrections originate from Loop Quantum Cosmology What comes to the fore are corrections due to what is called quantum holonomy, l. A comment about the quantum bounce. i.e. what is given by Dah-Wei Chiou, Li-Fang Li, [66] (2009) is that there is a branch matchup between a prior to a present set of Whee- 
ler De Witt equations for a prior to present universe, as far as modeling how the quantum bounce links the two Wheeler De Witt solution branches, i.e. one Wheeler De Witt wave function for a prior univers, and another wave function for a present universe. Furthermore, Abhay Ashtekar [67] (2006) wrote a simple treatment of the Bounce causing Wheeler De Witt equation along the lines of, for $\rho_{*} \approx$ const $\cdot(1 / 8 \pi G \Delta)$ as a critical density, and $\Delta$ the eignvalue of a minimum area operator. Small values of $\Delta$ imply that gravity is a repulsive force, leading to a bounce effect.

$$
\left(\frac{\dot{a}}{a}\right)^{2} \equiv \frac{8 \pi G}{3} \cdot \rho \cdot\left(1-\left(\rho / \rho_{*}\right)\right)+\text { H.O.T. }
$$

Furthermore, Bojowald [68] (2008) specified a criteria as to how to use an updated version of $\Delta$ and $\rho_{*} \approx$ const $\cdot(1 / 8 \pi G \Delta)$ in his GRG manuscript on what could constitute grounds for the existence of generalized squeezed initial (graviton?) states. Bojowald (2008) was referring to the existence of squeezed states, as either being necessarily, or NOT necessarily a consequence of the quantum bounce. As Bojowald [68] (2008) wrote it up, in both his Equation (26) which has a quantum Hamiltonian $\langle\hat{V}\rangle \approx H$, with

$$
\left.\frac{\mathrm{d}\langle\hat{V}\rangle}{\mathrm{d} \phi}\right|_{\phi \approx 0} \underset{\text { existence-of-un-squeezed-states } \Leftrightarrow \phi \approx 0}{\longrightarrow} 0
$$

and $\hat{V}$ is a "volume" operator where the "volume" is set as $V$, Note also, that Bojowald [68] has in his initial Friedman equation, density values $\rho \equiv \frac{H_{\text {matter }}(a)}{a^{3}}$, so that when the Friedman equation is quantized, with an initial internal time given by $\phi$, with $\phi$ becoming a more general evolution of state variable than "internal time". If so, Bojowald [68] (2008) writes, when there are squeezed states

$$
\left.\frac{\mathrm{d}\langle\hat{V}\rangle}{\mathrm{d} \phi}\right|_{\phi \neq 0} \underset{\text { existence-of-squeezed-states }}{\longrightarrow} N(\text { value }) \neq 0
$$

for his Equation (26), which is incidently when links to classical behavior break down, and when the bounce from a universe contracting goes to an expanding present universe. Bojowald [68] also writes that if one is looking at an isotropic universe, that as the large matter " $\mathrm{H}$ " increases, that in certain cases, one observes more classical behavior, and a reduction in the strength of a quantum bounce.. Bojowalds [68] states that "Especially the role of squeezed states is highlighted. The presence of a bounce is proven for uncorrelated states, but as squeezing is a dynamical property and may change in time" The upshot is that although it is likely in a quantum bounce state that the states should be squeezed, it is not a pre requisite for the states to always start off as being squeezed states. So a physics researcher can, look at if an embedding of the present universe in a higher dimensional structure which could have lead to a worm hole from a prior universe to our present for re introduction of inflationary growth.

F. Other models. Do worm hole bridges between different universes allow for initial un squeezed states? Wheeler De Witt solution with pseudo time component added in. 
This discussion is to present a not so well known but useful derivation of how instant on structure from a prior universe may be transferred from a prior to the present universe.

1. The solution as taken from L. Crowell's (2005) [69] book, and re produced here, as referenced by Beckwith $(2008,2009)$ has many similarities with the WKB method. i.e. it is semi CLASSICAL.

2. left unsaid is what embedding structure is assumed

3. A final exercise for the reader. Would a WKB style solution as far as transfer of "material" from a prior to a present universe constitute procedural injection of non compressed states from a prior to a present universe? Also if uncompressed, coherent states are possible, how long would they last in introduction to a new universe?

This is the Wheeler-De-Witt equation with pseudo time component added. From Crowell [69]

$$
-\frac{1}{\eta r} \frac{\partial^{2} \Psi}{\partial r^{2}}+\frac{1}{\eta r^{2}} \cdot \frac{\partial \Psi}{\partial r}+r R^{(3)} \Psi=(r \eta \phi-r \ddot{\phi}) \cdot \Psi
$$

This has when we do it $\phi \approx \cos (\omega \cdot t)$, and frequently $R^{(3)} \approx$ constant, so then we can consider

$$
\phi \cong \int_{0}^{\infty} \mathrm{d} \omega\left[a(\omega) \cdot \mathrm{e}^{i k_{\sigma} x^{\mu}}-a^{+}(\omega) \cdot \mathrm{e}^{-i k_{\omega} x^{\mu}}\right]
$$

In order to do this, we can write out the following for the solutions to Equation (67) above [69].

$$
\begin{aligned}
C_{1}= & \eta^{2} \cdot\left(4 \cdot \sqrt{\pi} \cdot \frac{t}{2 \omega^{5}} \cdot J_{1}(\omega \cdot r)+\frac{4}{\omega^{5}} \cdot \sin (\omega \cdot r)+(\omega \cdot r) \cdot \cos (\omega \cdot r)\right) \\
& +\frac{15}{\omega^{5}} \cos (\omega \cdot r)-\frac{6}{\omega^{5}} \operatorname{Si}(\omega \cdot r)
\end{aligned}
$$

And

$$
C_{2}=\frac{3}{2 \cdot \omega^{4}} \cdot(1-\cos (\omega \cdot r))-4 e^{-\omega \cdot r}+\frac{6}{\omega^{4}} \cdot \operatorname{Ci}(\omega \cdot r)
$$

This is where $\operatorname{Si}(\omega \cdot r)$ and $C i(\omega \cdot r)$ refer to integrals of the form $\int_{-\infty}^{x} \frac{\sin \left(x^{\prime}\right)}{x^{\prime}} \mathrm{d} x^{\prime}$ and $\int_{-\infty}^{x} \frac{\cos \left(x^{\prime}\right)}{x^{\prime}} d x^{\prime}$. Next, we should consider whether or not the instant on so formed is stable under evolution of space-time leading up to inflation. To model this, we use results from Crowell (2005) [69] on quantum fluctuations in space-time, which gives a model from a pseudo time component version of the Wheeler-De-Witt equation, with use of the Reinssner-Nordstrom metric to help us obtain a solution that passes through a thin shell separating two space-times. The radius of the shell $r_{0}(t)$ separating the two space-times is of length $l_{P}$ in approximate magnitude, leading to a domination of the time component for the Reissner-Nordstrom metric [69]

$$
\mathrm{d} S^{2}=-F(r) \cdot \mathrm{d} t^{2}+\frac{\mathrm{d} r^{2}}{F(r)}+\mathrm{d} \Omega^{2}
$$


This has:

$$
F(r)=1-\frac{2 M}{r}+\frac{Q^{2}}{r^{2}}-\frac{\Lambda}{3} \cdot r^{2} \underset{T \rightarrow 10^{32} \text { Kelvin } \sim \infty}{\longrightarrow}-\frac{\Lambda}{3} \cdot\left(r=l_{P}\right)^{2}
$$

This assumes that the cosmological vacuum energy parameter has a temperature dependence as outlined by Park [70] (2003), leading to

$$
\frac{\partial F}{\partial r} \sim-2 \cdot \frac{\Lambda}{3} \cdot\left(r \approx l_{P}\right) \equiv \eta(T) \cdot\left(r \approx l_{P}\right)
$$

as a wave functional solution to a Wheeler-De-Witt equation bridging two space-times, similar to two space-times with "instantaneous" transfer of thermal heat, as given by Crowell (2005) [69]

$$
\Psi(T) \propto-A \cdot\left\{\eta^{2} \cdot C_{1}\right\}+A \cdot \eta \cdot \omega^{2} \cdot C_{2}
$$

This has $C_{1}=C_{1}(\omega, t, r)$ as a pseudo cyclic and evolving function in terms of frequency, time, and spatial function. This also applies to the second cyclical wave function $C_{2}=C_{2}(\omega, t, r)$, where $C_{1}=$ Equation (69) and $C_{2}=$ Equation (70); Equation (74) is a solution to the pseudo time WDM equation.

The question which will be investigated is if Equation (74) is a way to present either a squeezed or un squeezed state. A way forward is to note that Prado Martin-Moruno, Pedro F. Gonzalez-Diaz in [71] July (2009) wrote up about thermal phantom-like radiation process coming from the wormhole throat. Note that the Crowell construction of a worm hole bridge is in some ways similar to Carco Cavaglià's [72] (1994) treatment of use of conjugate momentum $\pi^{i j}$ of $h_{i j}$ generalized momentum variables, also known as conjugate momenta $\hat{\pi}^{i j} \equiv \frac{\hbar}{i} \cdot \frac{\partial}{\partial \cdot h_{i j}}$, leading to the sort of formalism as attributed to Luis J. Garay's [73] (1991) article, of

$$
\Psi\left(h_{i j}\right) \approx\left[\exp \int \mathrm{d}^{3} x \cdot \pi^{i j} \cdot h_{i j}\right]_{T}
$$

Now in the case of what can be done with the worm hole used by Crowell, with, if $\hbar \equiv 1, \quad \hat{\pi}^{i j} \equiv-i \frac{\delta}{\delta \cdot g_{i j}}, \quad \hat{\pi}^{\theta \theta} \equiv-\frac{i}{2 r} \frac{\partial}{\partial r}, \quad \hat{\pi}^{t t} \equiv-i \cdot\left(\frac{\partial F(r)}{\partial r}\right)^{-1} \cdot \frac{\partial}{\partial r}$, and a kinetic energy value as given of the form $\hat{\pi}^{\theta \theta} \hat{\pi}^{t t}+\hat{\pi}^{t t} \hat{\pi}^{\theta \theta}$. The supposition which we have the worm hole wave functional may be like, so, use the wave functional looking like $\Psi\left(g_{i j}\right) \approx\left[\exp \int \mathrm{d}^{3} x \cdot\left[\pi^{i j}\right] \cdot g_{i j}\right]_{T}$ where the $g_{i j}$ for the Weiner-Nordstrom metric will be

$$
\begin{aligned}
\mathrm{d} S^{2} & =-F(r) \cdot \mathrm{d} t^{2}+\frac{\mathrm{d} r^{2}}{F(r)}+\mathrm{d} \Omega^{2} \\
& \equiv g^{i j} \mathrm{~d} x^{i} \mathrm{~d} x^{j} \equiv \frac{\Lambda}{3} \cdot\left(r=l_{P}\right)^{2} \cdot \mathrm{d} t^{2}-\frac{\mathrm{d} r^{2}}{\frac{\Lambda}{3} \cdot\left(r=l_{P}\right)^{2}}+\mathrm{d} \Omega^{2}
\end{aligned}
$$

G. Creating an analysis of how graviton mass, assuming branes, can influence expansion of the universe

Following development of ${ }^{\star}(1.13)$ as mentioned above, with inputs from Friedman eqns. To do this,, the following normalizations will be used, i.e. $\hbar=c=1$, so then 


$$
q=A 1+A 2+A 3+A 4
$$

where

$$
\begin{gathered}
A 1=\frac{C}{a^{3}} \cdot\left[1 / \sqrt{\frac{C}{a^{4}}-\frac{\kappa}{a^{2}}+\left(\frac{\rho}{3 M_{4}^{2}}+\frac{\Lambda_{4}}{3}+\frac{1}{36} \cdot \frac{\rho^{2}}{M_{P}^{6}}\right)}\right] \\
A 1=\frac{C}{a^{3}} \cdot\left[1 / \sqrt{\frac{C}{a^{4}}-\frac{\kappa}{a^{2}}+\left(\frac{\rho}{3 M_{4}^{2}}+\frac{\Lambda_{4}}{3}+\frac{1}{36} \cdot \frac{\rho^{2}}{M_{P}^{6}}\right)}\right] \\
A 2=-\left(\frac{\rho}{3 M_{4}^{2}}+\frac{\Lambda_{4}}{3}+\frac{1}{36} \cdot \frac{\rho^{2}}{M_{P}^{6}}\right) /\left[\frac{C}{a^{4}}-\frac{\kappa}{a^{2}}+\left(\frac{\rho}{3 M_{4}^{2}}+\frac{\Lambda_{4}}{3}+\frac{1}{36} \cdot \frac{\rho^{2}}{M_{P}^{6}}\right)\right]
\end{gathered}
$$

$A 3=$

$$
\begin{gathered}
-\frac{1}{2} \cdot\left[\frac{(\mathrm{d} \rho / \mathrm{d} \tau)}{3 M_{4}^{2}}+\frac{\left(\mathrm{d} \Lambda_{4} / \mathrm{d} \tau\right)}{3}+\frac{1}{18} \cdot \frac{\rho \cdot(\mathrm{d} \rho / \mathrm{d} \tau)}{M_{P}^{2}}\right] /\left[\frac{C}{a^{4}}-\frac{\kappa}{a^{2}}+\left(\frac{\rho}{3 M_{4}^{2}}+\frac{\Lambda_{4}}{3}+\frac{1}{36} \cdot \frac{\rho^{2}}{M_{P}^{6}}\right)\right]^{3 / 2} \\
A 4=\frac{\kappa}{a^{3}} \cdot\left[\frac{(\mathrm{d} a / \mathrm{d} \tau)}{3}\right] /\left[\frac{C}{a^{4}}-\frac{\kappa}{a^{2}}+\left(\frac{\rho}{3 M_{4}^{2}}+\frac{\Lambda_{4}}{3}+\frac{1}{36} \cdot \frac{\rho^{2}}{M_{P}^{6}}\right)\right]^{3 / 2}
\end{gathered}
$$

Furthermore, if we are using density according to whether or not 4 dimensional graviton mass is used, then

$$
\rho \equiv \rho_{0} \cdot\left(\frac{a_{0}}{a}\right)^{3}-\left[\frac{m_{g} c^{6}}{8 \pi G \hbar^{2}}\right] \cdot\left(\frac{a^{4}}{14}+\frac{2 a^{2}}{5}-\frac{1}{2}\right)
$$

So, then one can look at $\mathrm{d} \rho / \mathrm{d} \tau$ obtaining

$$
\mathrm{d} \rho / \mathrm{d} \tau=-\left(\frac{\dot{a}}{a}\right) \cdot\left[3 \cdot \rho_{0} \cdot\left(\frac{a_{9}}{a}\right)^{3}+4 \cdot\left(\frac{a^{4}}{14}+\frac{a^{2}}{5}\right) \cdot\left(\frac{m_{g} c^{6}}{8 \pi G \hbar^{2}}\right)\right]
$$

Here, use,

$$
\left(\frac{\dot{a}}{a}\right)=\sqrt{\frac{C}{a^{4}}-\frac{\kappa}{a^{2}}+\left(\frac{\rho}{3 M_{4}^{2}}+\frac{\Lambda_{4}}{3}+\frac{1}{36} \cdot \frac{\rho^{2}}{M_{P}^{6}}\right)}
$$

and

If $\hbar \equiv c \equiv 1$,

$\mathrm{d} \rho / \mathrm{d} \tau=-\sqrt{\frac{C}{a^{4}}-\frac{\kappa}{a^{2}}+\left(\frac{\rho}{3 M_{4}^{2}}+\frac{\Lambda_{4}}{3}+\frac{1}{36} \cdot \frac{\rho^{2}}{M_{P}^{6}}\right)} \cdot\left[3 \cdot \rho_{0} \cdot\left(\frac{a_{9}}{a}\right)^{3}+4 \cdot\left(\frac{a^{4}}{14}+\frac{a^{2}}{5}\right) \cdot\left(\frac{m_{g}}{8 \pi G}\right)\right]$

Now, if, to first order, $\mathrm{d} \Lambda_{4} / \mathrm{d} \tau \sim 0$, and also, we neglect $\Lambda_{4}$ as of being not a major contributor

$$
\begin{aligned}
& \mathrm{d} \rho / \mathrm{d} \tau \cong-\sqrt{\frac{C}{a^{4}}-\frac{\kappa}{a^{2}}+\left(\frac{\rho}{3 M_{4}^{2}}+\frac{1}{36} \cdot \frac{\rho^{2}}{M_{P}^{6}}\right)} \cdot\left[3 \cdot \rho_{9} \cdot\left(\frac{a_{0}}{a}\right)^{3}+4 \cdot\left(\frac{a^{4}}{14}+\frac{a^{2}}{5}\right) \cdot\left(\frac{m_{g}}{8 \pi G}\right)\right] \\
& A 3 \cong \frac{1}{2}\left(\left[\frac{1}{3 M_{4}^{2}}+\frac{1}{18} \cdot \frac{\rho}{M_{P}^{6}}\right] /\left[\frac{C}{a^{4}}-\frac{\kappa}{a^{2}}+\left(\frac{\rho}{3 M_{4}^{2}}+\frac{1}{36} \cdot \frac{\rho^{2}}{M_{P}^{6}}\right)\right]^{1 / 2}\right) \\
& \cdot\left[3 \cdot \rho_{0} \cdot\left(\frac{a_{0}}{a}\right)^{3}+4 \cdot\left(\frac{a^{4}}{14}+\frac{a^{2}}{5}\right) \cdot\left(\frac{m_{g}}{8 \pi G}\right)\right] \text {. }
\end{aligned}
$$


Also, then, set the curvature equal to zero. i.e. $\kappa=0$, so then $A 4=0$, and

$$
\begin{aligned}
A 3 \cong & \frac{1}{2}\left(\left[\frac{1}{3 M_{4}^{2}}+\frac{1}{18} \cdot \frac{\rho \cdot}{M_{P}^{6}}\right] /\left[\frac{C}{a^{4}}+\left(\frac{\rho}{3 M_{4}^{2}}+\frac{1}{36} \cdot \frac{\rho^{2}}{M_{P}^{6}}\right)\right]^{1 / 2}\right) \\
& \cdot\left[3 \cdot \rho_{0} \cdot\left(\frac{a_{0}}{a}\right)^{3}+4 \cdot\left(\frac{a^{4}}{14}+\frac{a^{2}}{5}\right) \cdot\left(\frac{m_{g}}{8 \pi G}\right)\right] .
\end{aligned}
$$

Then

$$
\begin{gathered}
A 2 \cong-\left(\frac{\rho}{3 M_{4}^{2}}+\frac{1}{36} \cdot \frac{\rho^{2}}{M_{P}^{6}}\right) /\left[\frac{C}{a^{4}}+\left(\frac{\rho}{3 M_{4}^{2}}+\frac{1}{36} \cdot \frac{\rho^{2}}{M_{P}^{6}}\right)\right] \\
A 1 \cong \frac{C}{a^{3}} \cdot\left[1 / \sqrt{\frac{C}{a^{4}}+\left(\frac{\rho}{3 M_{4}^{2}}+\frac{1}{36} \cdot \frac{\rho^{2}}{M_{P}^{6}}\right)}\right]
\end{gathered}
$$

Pick, here,

$$
\rho \equiv \rho_{0} \cdot\left(\frac{a_{0}}{a}\right)^{3}-\left[\frac{\mathrm{m}_{\mathrm{g}}}{8 \pi \mathrm{G}}\right] \cdot\left(\frac{a^{4}}{14}+\frac{2 a^{2}}{5}-\frac{1}{2}\right)
$$

after $\hbar=c=1$, and also set

$$
\begin{gathered}
\Phi(\rho, a, C)=\frac{C}{a^{4}}+\left(\frac{\rho}{3 M_{4}^{2}}+\frac{1}{36} \cdot \frac{\rho^{2}}{M_{P}^{6}}\right) \\
A 3 \cong \frac{1}{2}\left(\left[\frac{1}{3 M_{4}^{2}}+\frac{1}{18} \cdot \frac{\rho}{M_{P}^{6}}\right] /[\Phi(\rho, a, C)]^{1 / 2}\right) \cdot\left[3 \cdot \rho_{0} \cdot\left(\frac{a_{0}}{a}\right)^{3}+4 \cdot\left(\frac{a^{4}}{14}+\frac{a^{2}}{5}\right) \cdot\left(\frac{m_{g}}{8 \pi G}\right)\right] \\
A 2 \cong-\left(\frac{\rho}{3 M_{4}^{2}}+\frac{1}{36} \cdot \frac{\rho^{2}}{M_{P}^{6}}\right) /[\Phi(\rho, a, C)] \\
A 1 \cong \frac{C}{a^{3}} \cdot[1 / \sqrt{\Phi(\rho, a, C)}]
\end{gathered}
$$

For what it is worth, the above can have the shift to red shift put in by the following substitution. i.e. use $1+z=a_{0} / a$. Assume also that $C$ is the dark radiation term which in the brane version of the Friedman equation scales as $a^{-4}$ and has no relationship to the speed of light. $a_{0}$ is the value of the scale factor in the present era, when red shift $z=0$, and $a \equiv a(\tau)$ in the past era, where $\tau$ is an interval of time after the onset of the big bang. $\left(a_{0} / a\right)^{3}=(1+z)^{3}$, and $a \equiv a_{0} /(1+z)$, Then

$$
\begin{gathered}
\rho(z) \equiv \rho_{0} \cdot(1+z)^{3}-\left[\frac{m_{g}}{8 \pi G}\right] \cdot\left(\frac{a_{0}^{4}}{14 \cdot(1+z)^{4}}+\frac{2 a_{0}^{2}}{5 \cdot(1+z)^{2}}-\frac{1}{2}\right) \\
A 1(z) \cong \frac{C \cdot(1+z)^{3}}{a_{0}^{3}} \cdot\left[1 / \sqrt{\Phi\left(\rho(z), a_{0} /(1+z), C\right)}\right] \\
A 2(z) \cong-\left(\frac{\rho(z)}{3 M_{4}^{2}}+\frac{1}{36} \cdot \frac{\rho(z)^{2}}{M_{P}^{6}}\right) /\left[\Phi\left(\rho(z), a_{0} /(1+z), C\right)\right]
\end{gathered}
$$




$$
\begin{aligned}
& A 3(z) \cong \frac{1}{2}\left(\left[\frac{1}{3 M_{4}^{2}}+\frac{1}{18} \cdot \frac{\rho(z)}{M_{P}^{6}}\right] /\left[\Phi\left(\rho(z), a_{0} /(1+z), C\right)\right]^{1 / 2}\right) \\
& \cdot\left[3 \cdot \rho_{0} \cdot(1+z)^{3}+4 \cdot\left(\frac{a_{0}^{4} /(1+z)^{4}}{14}+\frac{a_{0}^{2} /(1+z)^{2}}{5}\right) \cdot\left(\frac{m_{g}}{8 \pi G}\right)\right] \\
& \Phi\left(\rho(z), a_{0} /(1+z), C\right)=\frac{C \cdot(1+z)^{4}}{a_{0}^{4}}+\left(\frac{\rho(z)}{3 M_{4}^{2}}+\frac{1}{36} \cdot \frac{\rho(z)^{2}}{M_{P}^{6}}\right)
\end{aligned}
$$

So, for $4<z \leq 0$, i.e. not for the range, say $z \sim 1100380$ thousand years after the big bang, it would be possible to model, here

$$
q(z)=A 1(z)+A 2(z)+A 3(z)
$$

Easy to see though, that to first order, $q(z)=A 1(z)+A 2(z)+A 3(z)$ would be enormous when $z \sim 1100$, and also that for $Z=0, q(0)=A 1(0)+A 2(0)+A 3(0)>0$. Negative values for Equation (2.23) appear probable at about $z \sim 1.5$, when Equation (98) would dominate, leading to $q(z \sim 1.5)$ with a negative expression/value. The positive value conditions rely upon, the $C$ dark radiation term

\section{Conclusions: Unanswered Questions, and What This Suggests for Future Research Endeavors}

As far back as 1982 [74], Linde, when analyzing a potential of the form

$$
V(\phi)=\frac{m^{2} \phi^{2}}{2}+\lambda \phi^{4}+V(0)
$$

This is when the "mass" has the form, (here $\mathrm{M}$ is the bare mass term of the field $\phi$ in de Sitter space, which does not take into account quantum fluctuations)

$$
m^{2}(t)=M^{2}+\frac{3 \lambda H^{3}}{4 \pi} \cdot\left(t-t_{0}\right)
$$

Specified non linearity of $\left\langle\phi^{2}\right\rangle$ at a time from the big bang, of the form

$$
\Delta t_{1} \approx \frac{3 H}{2 M}
$$

The question raised repeatedly in whether or not i) if higher dimensions are necessary, and whether or not ii) mass gravitons are playing a role as far as the introduction of DE speed up of cosmological expansion may lead to an improvement over what was specified for density fluctuations and structure formation (the galaxy hierarchy problem) of density fluctuations given as

$$
\frac{\delta \rho}{\rho} \sim 10^{-4} \Leftrightarrow \lambda \leq 10^{-10}
$$

Equation (105) is for four space, a defining moment as to what sort of model would lead to density fluctuations. It totally fails as to give useful information as to the galaxy hierarchy problem as given in Figure 1, above. Secondly, to what degree is the relative speed up of the $q(z)$ function is impacted by various inter plays between, say a MODIFIED version of, say a KK DM model, using a MODIFIED mass hierarchy to get suitable DM masses of the order of $100 \mathrm{GeV}$ or more. Giving a suitable definition as to $q(z)$ as well as the inter play between DM values, 4 Dim Graviton mass issues, and/or 
what really contributes to the speed up of the universe will in the end dramatically improve the very crude estimate given by Equation (105) above which says next to nothing about how the problems illustrated by the breakdown of the galaxy mass formation/ hierarchy can be fixed. Furthermore is considering the spectral index problem, where the spectral index is [74]

$$
n_{S}-1 \cong-\frac{3}{8 \pi} \cdot\left(\frac{V_{\phi}}{V}\right)^{2}+\frac{1}{4 \pi} \cdot\left(\frac{V_{\phi \phi}}{V}\right)^{2}
$$

Usual experimental values of density fluctuations experimentally are $\frac{\delta \rho}{\rho} \sim 10^{-5}$, instead of $\frac{\delta \rho}{\rho} \sim 10^{-4}$, and this is assuming that $\lambda$ is extremely small. In addition, Linde (1982) [14] had $\frac{\mathrm{d}}{\mathrm{d} \phi^{2}} V=m^{2} \leq \frac{H}{40}=\frac{1}{40} \cdot \frac{\dot{a}}{a}$ inside a false vacuum bubble. If something other than the Klein Gordon relationship $\frac{\dot{a}}{a} \Rightarrow \ddot{\phi}+3 H \dot{\phi}+m^{2} \varphi=0$ occurs, then different models of how density fluctuation may have to be devised. A popular model of density fluctuations with regards to the horizon is

$$
\left(\frac{\delta \rho}{\rho}\right)_{\text {Horizon }} \cong \frac{k^{3 / 2}\left|\delta_{k}\right|}{\sqrt{2} \pi} \propto \frac{k^{(3 / 2)+3 \alpha-3 / 2}}{\sqrt{2} \pi} \approx(1 / \sqrt{2} \pi) \cdot k^{3 \alpha}
$$

where $-0.1<\alpha<0.2$, and $\alpha \equiv 0 \Leftrightarrow n_{s} \equiv 1$ and to first order, $k \cong H a$. The values, typically of $n_{s} \neq 1$ If working with $H^{2}=\left(\frac{\dot{a}}{a}\right)^{2}=\left[\left(\frac{\rho}{3 M_{4}^{2}}+\frac{\rho^{2}}{36 M_{\text {Planck }}^{2}}\right)+\frac{C}{a^{4}}\right]$, and with a density value $\rho \equiv \rho_{0} \cdot\left(\frac{a_{0}}{a}\right)^{3}-\left[\frac{m_{g} c^{6}}{8 \pi G \hbar^{2}}\right] \cdot\left(\frac{a^{4}}{14}+\frac{2 a^{2}}{5}-\frac{1}{2}\right)$ where $m_{g} \approx 10^{-65}$ grams, and $\alpha<0.2$ is usually picked to avoid over production of black holes, a very complex picture emerges. Furthermore, if working with $\alpha<0.2$ and $\alpha \neq 0$

$$
\left(\frac{\delta \rho}{\rho}\right)_{\text {Horizon }} \cong(1 / \sqrt{2} \pi) \cdot k^{3 \alpha} \sim \frac{H^{2}}{\dot{\phi}} \propto 10^{-4}-10^{-5}
$$

The above equation gives inter relationships between the time evolution of a pop up inflaton field $\phi$, and a Hubble expansion parameter $\mathrm{H}$, and a wave length parameter $\lambda=(2 \pi / k) \cdot a(t)$ for a mode given as $\delta_{k}$. What should be considered is the inter relationship of the constituent components of (3.6) and $\lambda \leq H^{-1}$. What the author thinks is of particular import is to look at whether or not the more general expression, as given by Steinhardt

$$
\left(\frac{\delta \rho}{\rho}\right) \cong A k^{\left(\frac{n_{S}-1}{2}\right)} \propto 10^{-4}-10^{-5}
$$

To first order, variations of $\alpha<0.2$ and $\alpha \neq 0$, should be compared with admissible values of $\left(\left[n_{S}-1\right] / 2\right)$ which would closely correspond to $\alpha \neq 0$ and $0<\alpha \ll 0.2$. i.e. the precise values of this may help us out in determining how to unravel what is going on in the galaxy formation picture as given in Figure 1 on page 6, break down. i.e. how can we have earlier than expected galaxy formation? 
The follow ups to the conclusion are THAT WE stress that the achievement of conditions allowing us to do research ON THE FUNDATIONS OF A WORKING gravitational wave astronomy research protocol, will be important for discriminating among general relativity and other gravity theories, as it has been shown in [75].

i.e. we need to understand what gravity is. That is basic.

Furthermore, the foundational issues brought up by the LIGO discovery should be confirmed and expanded upon as given in [76] and [77].

\section{Acknowledgements}

This work is supported in part by National Nature Science Foundation of China grant No. 11375279.

\section{References}

[1] Penrose, R. (2007) The Road to Reality: A Complete Guide to the Laws of the Universe. Vintage Books, New York.

[2] Wesson, P.S. (1999) Space-Time-Matter, Modern Kaluza-Klein Theory. World Scientific, Singapore. https://doi.org/10.1142/3889

[3] Polchinski, J. String Theory Volume 1: An Introduction to the Bosonic String. http://ebooks.cambridge.org/ebook.jsf?bid=CBO9780511816079

[4] Haisch, B., Rueda, A. and Dobyns, Y. (2001) Inertial Mass and the Quantum Vacuum Fields. Annalen der Physik, 10, 393-414. arXiv:gr-qc/0009036.

[5] Tong, M.-L., Zhang, Y. and Li, F.-Y. (2008) Using Polarized Maser to Detect HighFrequency Relic Gravitational Waves. Physical Review D, 78, Article ID: 024041.

http://arxiv.org/abs/0807.0885 https://doi.org/10.1103/PhysRevD.78.024041

[6] Beckwith, A.W. (2009) Entropy Growth in the Early Universe and Confirmation of Initial big Bang Conditions (Wheeler De Witt Eqn. Results vs. String Theory?). http://vixra.org/abs/0908.0109

[7] Ng, Y. (2008) Spacetime Foam: From Entropy and Holography to Infinite Statistics and Nonlocality. Entropy, 10, 441-461. https://doi.org/10.3390/e10040441

[8] Glinka, L. (2007) Quantum Information from Graviton-Matter Gas. Sigma, 3, 087, 13 p.

[9] Kolb, E. and Turner, S. (1994) The Early Universe. Westview Press, Chicago.

[10] Lloyd, S. (2002) Computational Capacity of the Universe. Physical Review Letters, 88, Article ID: 237901. https://doi.org/10.1103/PhysRevLett.88.237901

[11] Allen, B., Flanagan, E. and Papa, M.A. (2000) Is the Squeezing of Relic Gravitational Waves Produced by Inflation Detectable? Physical Review D, 61, Article ID: 024024.

http://arxiv.org/PS_cache/gr-qc/pdf/9906/9906054v2.pdf https://doi.org/10.1103/PhysRevD.61.024024

[12] Tong, M.-L. and Zhang, Y. (2007) Detecting Very-High-Frequency Relic Gravitational Waves by Electromagnetic Wave Polarizations in a Waveguide. Chinese Journal of Astronomy and Astrophysics, 8 (2008), 314-328. http://arxiv.org/abs/0711.4909 https://doi.org/10.1088/1009-9271/8/3/08

[13] Ingley, R.M.J. and Cruise, A.M. (2001) An Electromagnetic Detector for High Frequency Gravitational Waves. 4th Edoardo Amaldi Conference on Gravitational Waves, Perth, Australia, July 2001.

[14] Linder, E. (2003) Exploring the Expansion History of the Universe. Physical Review Letters, 
90, Article ID: 091301. https://doi.org/10.1103/PhysRevLett.90.091301

[15] Will, C. (1997) Bounding the Mass of the Graviton Using Gravitional-Wave Observations of Inspiralling Compact Binaries. http://arxiv.org/abs/gr-qc/9709011

[16] Sokolowski, L.M. and Staruszkiewicz, A. (2006) On the issue of Gravitons. Classical and Quantum Gravity, 23, 5907-5918. http://arxiv.org/abs/gr-qc/0606111 https://doi.org/10.1088/0264-9381/23/20/012

[17] Janssen, B. and Lozano, Y. (2003) A Microscopical Description of Giant Gravitons. Nuclear Physics B, 658, 281-299. https://arxiv.org/abs/hep-th/0207199 https://doi.org/10.1016/S0550-3213(03)00185-8

[18] Durrer, R. and Rinaldi, M. (2009) Graviton Production in Non-Inflationary Cosmology. Physical Review D, 79, Article ID: 063507. http://arxiv.org/abs/0901.0650 https://doi.org/10.1103/PhysRevD.79.063507

[19] Giovannini, M. (2008) A Primer on the Physics of the Cosmic Microwave Background. World Press Scientific, Singapore.

[20] Rubakov, V.A. Large and Infinite Extra Dimensions. http://arxiv.org/pdf/hep-ph/0104152.pdf

[21] Rubakov V. (2002) Classical Theory of Gauge Fields. Princeton University Press, Princeton, Translated by Stephen S. Wilson, $456 \mathrm{p}$.

[22] Rubakov, V.A. (2009) Baikal Summer School on Physics of Elementary Particles and Astrophysics; Talk Given in This Conference, i.e. Notes.

[23] Baumann, D., Ichiki, K., Steinhardt, P.J. Takahashi, K. Gravitational Wave Spectrum Induced by Primordial Scalar Perturbations. arXiv:hep-th/0703290

[24] Gregory, R. Rubakov, V.A. and Sibiryakov, S.M. (2000) Brane Worlds: The Gravity of Escaping Matter. Classical and Quantum Gravity, 17, 4437-4450.

https://arxiv.org/abs/hep-th/0003109 https://doi.org/10.1088/0264-9381/17/21/306

[25] Fedeli, C., Moscardini, L. and Matarrese, S. (2009) The Clustering of Galaxy Clusters in Cosmological Models with Non-Gaussian Initial Conditions: Predictions for Future Surveys. Monthly Notices of the Royal Astronomical Society, 397, 1125-1137.

http://arxiv.org/abs/0904.3248

https://archive.org/stream/arxiv-0904.3248/0904.3248_djvu.txt https://doi.org/10.1111/j.1365-2966.2009.15042.x

[26] Matarrese, S. (2009) Lecture, 10 July 2009. International School of Astroparticle Physics, Como.

[27] Beckwith, A. (2010) Entropy, Neutrino Physics, and the Lithium Problem: Why Are There Stars with Essentially No Lithium Due to Serious Lithium Deficiency in Certain Spatial Regions in the Early Universe? Progress in Particle and Nuclear Physics, 64, 426-428.

https://doi.org/10.1016/j.ppnp.2009.12.066

[28] Dubovsky, S., Flauger, R., Starobinsky, A. and Thackev, I. (2009) Signatures of a Graviton Mass in the Cosmic Microwave Background. Physical Review D, 81, Article ID: 023523, 2010. https://arxiv.org/abs/0907.1658 https://doi.org/10.1103/PhysRevD.81.023523

[29] Alves, M.E.S., Miranda, O.D. and de Araujo, J.C.N. (2009) Can Massive Gravitons Be an Alternative to Dark Energy? arXiv:0907.5190

[30] Jones, M.H. and Lambourne, R.J. (2004) An Introduction to Galaxies and Cosmology. Cambridge University Press, Cambridge, UK, 244.

[31] Bolotin, Yu.L., Cherkaskiy, V.A., Lemets O.A., Yerokhin, D.A. and Zazunov, L.G. Cosmology in Terms of the Deceleration Parameter. Part I. http://arxiv.org/pdf/1502.00811.pdf 
[32] Maartens, R. (2004) Brane-World Gravity. http://www.livingreviews.org/lrr-2004-7

[33] Grössing, G. From Classical Hamiltonian Flow to Quantum Theory: Derivation of the Schrödinger Equation. https://arxiv.org/ftp/quant-ph/papers/0311/0311109.pdf

[34] Beckwith, A. Identifying a Kaluza Klein Treatment of a Graviton Permitting a Deceleration Parameter Q(Z) As An Alternative to Standard DE. http://journalofcosmology.com/BeckwithGraviton.pdf

[35] Peskins, M. and Schroeder, D. (1995) An Introduction to Quantum Field Theory. Westview Press, Palo Alto.

[36] Clifton, T., Ferreira, P.G. and Land, K. (2008) Living in a Void: Testing the Copernican Principle with Distant Supernovae. Physical Review Letters, 101, Article ID: 131302. https://arxiv.org/abs/0807.1443 https://doi.org/10.1103/PhysRevLett.101.131302

[37] Verde, L. Non Gaussianity versus Non Linearity of Cosmological Perturbations. Proc. of the 15th Florida workshop in Nonlinear Astronomy and Physics. Annals of The New York Academy of Sciences as well as. http://arxiv.org/abs/astro-ph/0004341

[38] Chingabam, P. and Park, C. Statistical Nature of Non Gaussianity from Cubic Order Primoridal Perturbations: CMB Map Simulations and Genus Statistics. http://arxiv.org/PS_cache/arxiv/pdf/0908/0908.1696v1.pdf

[39] Lehners, J.-L. and Steinhardt, P.J. (2008) Non-Gaussian Density Fluctuations from Entropically Generated Curvature Perturbations in Ekpyrotic Models. Physical Review D, 77, Article ID: 063533. http://arxiv.org/abs/0712.3779 https://doi.org/10.1103/PhysRevD.77.063533

[40] Bardeen, J.M. (1980) Gauge-Invariant Cosmological Perturbations. Physical Review D, 22, 1882-1905. https://doi.org/10.1103/PhysRevD.22.1882

[41] White, M. and Hu, W. The Sach-Wolfe Effect. http://arxiv.org/PS_cache/astro-ph/pdf/9609/9609105v1.pdf

[42] Babich, D., Creminelli, P. and Zaldarriaga M. (2004) The Shape of Non-Gaussianities. CAP, 0408, 009. http://arxiv.org/abs/astro-ph/0405356

[43] Lyth, D.H. (2005) Generating Curvature Perturbations at the End of Inflation. JCAP, Article ID: 0511006. http://arxiv.org/abs/astro-ph/0510443 https://doi.org/10.1088/1475-7516/2005/11/006

[44] Wechsler, R.H. (2001) Dark Halo Merging and Galaxy Formation. Ph.D. Thesis. http://risa.stanford.edu/thesis.php

[45] NASA/CXC/ESO/P Rosati et al. http://chandra.harvard.edu/photo/2004/rdcs1252/

[46] Rachel Courtland. https://www.newscientist.com/article/dn16912-overweight-galaxies-force-fed-by-dark-matt er-tendrils/

[47] Björn Helmut Bastian Opitz aus Warburg. http://www.desy.de/ horns/Opitz_Diss_Druckversion_2014-09-23.pdf

[48] Hinshaw, G., et al. Nine-Year Wilkinson Microwave Anisotropy Probe (WMAP) Observations: Cosmological Parameter Results. http://arxiv.org/abs/1212.5226

[49] Hingsaw G. Presentation, in COMO, Italy, July 2009 at the ISAPP.

[50] Glauber, R. (1963) Coherent and Incoherent States of the Radiation Field. Physical Review, 131, 2766-2788. https://doi.org/10.1103/PhysRev.131.2766

[51] Veneziano, G. (1986) A Stringy Nature Needs Just Two Constants. Europhysics Letters, 2, 199. Proc. of Texas Superstring Workshop (1989). https://doi.org/10.1209/0295-5075/2/3/006

[52] Maggiore, M. The Algebraic Structure of the Generalized Uncertainty Principle. 
https://arxiv.org/pdf/hep-th/9309034.pdf

[53] Kiefer, C. (2007) Quantum Gravity. International Series of Monographs on Physics, (Hardcover), Oxford Science Publications, Oxford University Press.

[54] Mohaupt, T. (2003) Introduction to String Theory. Lecture Notes in Physics, 631, 173-251. https://arxiv.org/abs/hep-th/0207249

[55] Ford, L.H. Gravitons and Lightcone Fluctuations. http://arxiv.org/abs/gr-qc/9410047

[56] Venkataratnam, K.K. and Suresh, P.K. (2008) Density Fluctuations in the Oscillatory Phase of Nonclassical Inflaton in FRW Universe. International Journal of Modern Physics D, 17, 1991-2005. http://arxiv.org/abs/0712.3129 https://doi.org/10.1142/S0218271808013662

[57] Grishchuk, L.P. (1998) The Detectability of Relic (Squeezed) Gravitational Waves by Laser Interferometers. http://arxiv.org/abs/gr-qc/9810055,

[58] Grishchuk, L.P. (1993) Quantum Effects In Cosmology. Classical and Quantum Gravity, 10, 2449-2478. http://arxiv.org/abs/gr-qc/9302036 https://doi.org/10.1088/0264-9381/10/12/006

[59] Grishchuk, L.P. (1980) The Problem of Relic Gravitational Radiation. Soviet Physics Uspekhi, 23, 705-706.

[60] Grishchuk, L. (2008) Discovering Relic Gravitational Waves in Cosmic Microwave Background Radiation. http://arxiv.org/abs/0707.3319

[61] Dick, R. (2001) Heterotic String Dark Matter from the Graviton Multiplet. Physics Letters B, 510, 10-16. https://doi.org/10.1016/S0370-2693(01)00617-7

[62] Berkenstein (2004). http://uw.physics.wisc.edu/ strings/group/slides.04.fall/berenstein.pdf

[63] Rovelli, C. (2006) Graviton Propagator from Background-Independent Quantum Gravity. Physical Review Letters, 97, Article ID:151301. http://arxiv.org/abs/gr-qc/0508124 https://doi.org/10.1103/PhysRevLett.97.151301

[64] Montl, L. (2007). http://motls.blogspot.com/2007/08/thiemann-dittrich-discreteness-of-lqg.html

[65] Mielczarek, J. (2009) Tensor Power Spectrum with Holonomy Corrections in Loop Quantum Cosmology. Physical Review D, 79, Article ID: 123520. http://arxiv.org/abs/0902.2490 https://doi.org/10.1103/PhysRevD.79.123520

[66] Chiou, D.-W. and Li, L.-F. (2009) Loop Quantum Cosmology With higher Order Holonomy Corrections. Physical Review D, 80, Article ID: 043512. http://arxiv.org/abs/0907.0640 https://doi.org/10.1103/PhysRevD.80.043512

[67] Abhay Ashtekar (2006) http://cgpg.gravity.psu.edu/people/Ashtekar/articles/solvaynet.pdf

[68] Bojowald, M. (2008) Quantum Nature of Cosmological Bounces. General Relativity and Gravitation, 40, 2659-2683. http://arxiv.org/abs/0801.4001 https://doi.org/10.1007/s10714-008-0645-1

[69] Crowell, L. (2005) Quantum Fluctuations of Space Time. World Scientific Series in Contemporary Chemical Physics, Vol. 25, World Scientific, PTE, LTD, Singapore.

[70] Park, D.K., Kim, H. and Tamarayan, S. (2002) Nonvanishing Cosmological Constant of Flat Universe in Brane-World Scenario. Physics Letters B, 535, 5-10.

http://arxiv.org/abs/hep-th/0111081 https://doi.org/10.1016/S0370-2693(02)01729-X

[71] Martín-Moruno, P. and González-Díaz, P.F. (2009) Thermal Radiation from Lorentzian Traversable Wormholes. Physical Review D, 80, Article ID: 024007. https://arxiv.org/abs/0907.4055

[72] Cavaglià, M. (1994) Quantum Electromagnetic Wormholes and Geometrical Description of the Electric Charge. Physical Review D, 50, 5087-5092. http://arxiv.org/abs/gr-qc/9407030 
https://doi.org/10.1103/PhysRevD.50.5087

[73] Garay, L.J. (1991) Quantum State of Wormholes and Path Integral. Physical Review D, 44, 1059-1066. https://doi.org/10.1103/PhysRevD.44.1059

[74] Lemoine, M., Martin, J. and Peter, P., Eds. (2008) Linde in Article in Physics 738, Inflationary Cosmology. Springer Verlag, Berlin.

[75] Corda, C. (2009) Interferometric Detection of Gravitational Waves: The Definitive Test for General Relativity. International Journal of Modern Physics D, 18, 2275-2282.

arXiv:0905.2502 [gr-qc]

[76] Abbott, B.P., et al. (2016) (LIGO Scientific Collaboration and Virgo Collaboration) Observation of Gravitational Waves from a Binary Black Hole Merger. Physical Review Letters, 116, Article ID: 061102. https://physics.aps.org/featured-article-pdf/10.1103/PhysRevLett.116.061102

[77] Abbott, B. P., et al. (LIGO Scientific Collaboration and Virgo Collaboration) (2016) GW151226: Observation of Gravitational Waves from a 22-Solar-Mass Binary Black Hole Coalescence. Physical Review Letters, 116, Article ID: 241103.

https://doi.org/10.1103/PhysRevLett.116.241103

\section{Submit or recommend next manuscript to SCIRP and we will provide best service} for you:

Accepting pre-submission inquiries through Email, Facebook, LinkedIn, Twitter, etc. A wide selection of journals (inclusive of 9 subjects, more than 200 journals)

Providing 24-hour high-quality service

User-friendly online submission system

Fair and swift peer-review system

Efficient typesetting and proofreading procedure

Display of the result of downloads and visits, as well as the number of cited articles

Maximum dissemination of your research work

Submit your manuscript at: http://papersubmission.scirp.org/

Or contact jhepgc@scirp.org 